\title{
Instability of meridional axial system in $f(R)$ gravity
}

\author{
M. Sharif ${ }^{a}$, Z. Yousaf ${ }^{b}$ \\ Department of Mathematics, University of the Punjab, Quaid-e-Azam Campus, Lahore 54590, Pakistan
}

Received: 8 April 2015 / Accepted: 15 April 2015 / Published online: 6 May 2015

(C) The Author(s) 2015. This article is published with open access at Springerlink.com

\begin{abstract}
We analyze the dynamical instability of a nonstatic reflection axial stellar structure by taking into account the generalized Euler equation in metric $f(R)$ gravity. Such an equation is obtained by contracting the Bianchi identities of the usual anisotropic and effective stress-energy tensors, which after using a radial perturbation technique gives a modified collapse equation. In the realm of the $R+\epsilon R^{n}$ gravity model, we investigate instability constraints at Newtonian and post-Newtonian approximations. We find that the instability of a meridional axial self-gravitating system depends upon the static profile of the structure coefficients, while $f(R)$ extra curvature terms induce the stability of the evolving celestial body.
\end{abstract}

\section{Introduction}

General relativity (GR) is a remarkable effort in mathematical physics to analyze gravitational effects of stellar relativistic interiors. Several interesting consequences coming from cosmic microwave background, observational ingredients of supernovae Ia and its cross-juxtaposition with foreground stellar galactic distributions [1-3] have made a revolution, thereby introducing a new research window. In this realm, many astrophysicists found GR modifications helpful to explore unknown aspects of cosmic gravitational dynamics. $f(R)$ gravity $[4,5]$ is among the extended gravity theories obtained by replacing the Ricci invariant with a generic function $f(R)$ of it in the Einstein-Hilbert action.

Anisotropic effects are leading paradigms in addressing the evolutionary mechanisms of celestial imploding models. Herrera and Santos [6] reviewed contributions of locally anisotropic fluid arrangements on the dynamical phases of collapsing shear and shear-free compact objects. Di Prisco et al. [7] investigated the dynamical properties of an

\footnotetext{
${ }^{\mathrm{a} e}$-mail: msharif.math@pu.edu.pk

be-mail: zeeshan.math@pu.edu.pk
}

anisotropic spherical matter distribution and found that small fluctuations of the pressure anisotropy lead to system cracking. Sharif et al. [8-15] analyzed the effects of anisotropy on the dynamical properties of spherical as well as non-spherical dense relativistic distributions and found very complicated system phases due to the presence of anisotropy. Sunzu et al. [16] studied analytical models of spherical anisotropic interiors and found that anisotropic effects provide a broader platform to discuss various forms of stellar relativistic systems. Recently, we [17-19] explored the dynamical features of anisotropic relativistic interiors.

The spinning stellar distributions indicate the direct relevance of the anisotropy for the gravitational evolution in which gravitational radiation cause vorticity within the observer congruences. Vorticity represents rotation of a neighboring fluid about an observer moving with relativistic matter distributions relative to an inertial frame. Herrera et al. [20] argued that such vorticity originates from the existence of a super-energy flow, which may have direct relevance in terms of the super-Poynting vector. Bonnor [21] found the electromagnetic energy flow in a relativistic compact distribution by formulating a relationship between the super-Poynting vector and vorticity. Korunur et al. [22] calculated various kinematical variables, like angular momentum, energy, and momentum of matter configurations associated with an axially symmetric scalar field. Li [23] explored the superradiant instability of rotating compact relativistic objects in higher dimensional theory and found configurations unstable against scalar field perturbations. Recently, Herrera et al. [24] presented a formal analysis of gravitational radiation within an anisotropic non-static reflection axial symmetric source and the existence of a super-energy flow linked with the matter vorticity.

A stability analysis of self-gravitating stellar systems in GR as well as modified gravity has attracted many researchers for the last few years. The study of different collapsing celestial models with extra degrees of freedom has great significance in the exploration of late-time cosmological evolu- 
tion. Chandrasekhar [25] discussed instability constraints for spherical symmetric relativistic geometry coupled with ideal matter configurations using the ratio of specific heats known as the stiffness parameter, $\Gamma_{1}$. Herrera et al. [26] investigated stability regions for radiating collapsing stellar objects and concluded that the dissipation vector tends to move the systems toward stable configurations. Chan et al. [27-29] studied the remarkable effects of the shearing viscosity and anisotropy on the instability constraints in the Newtonian (N) and post-Newtonian ( $\mathrm{pN}$ ) eras.

Cai [30] discussed the dynamical properties and the structure formation of dense matter relativistic configurations in modified gravity by assigning zero, negative, and positive values to the constant curvature. Bamba et al. [31] performed a dynamical analysis of a collapsing relativistic stellar system and claimed that invoking of $R^{\alpha}(1<\alpha \leq 2)$ corrections could help to construct a viable and singularity free model. Myung et al. [32] performed a stability analysis of the sphericalstellar structure with constant Ricci invariant background in metric $f(R)$ gravity via a perturbation scheme and noticed the relatively stable distributions under specific constraints. Moon et al. [33] extended these consequences to a negative cosmological constant environment and calculated the limits for the stability of relativistic systems.

Capozziello et al. [34] explored the dynamical evolution of relativistic collapsing spherical interior in $f(R)$ gravity by evaluating an extended form of the Poisson and Boltzmann equations. De Laurentis and Capozziello [35] discussed the instability issue of a stellar interior at the $\mathrm{N}$ approximation with $f(R)$ extra degrees of freedom and also studied axisymmetric black hole models. Astashenok et al. [36] investigated the evolution of self-gravitating systems and found relatively more massive and supergiant dense configurations due to $f(R)$ gravity corrections. Farinelli et al. [37] discussed the dynamical properties of stellar systems in the presence of $f(R)$ corrections and found that higher degree terms tend to mollify the collapsing process. Sharif et al. $[38,39]$ studied the instability constraints for a restricted class of an axially symmetric spacetime by means of the adiabatic index/stiffness parameter.

The present paper aims to extend our previous work [39] of stability analysis by taking reflection effects in non-static axial symmetric anisotropic source with $\epsilon R^{n}$ extra degrees of freedom. In the present paper, we develop instability regions for an anisotropic meridional axisymmetric source with a $R+\epsilon R^{n}$ background. The inclusion of an $\epsilon R^{n}$ correction in our analysis comes from the fact that this corresponds to the various eras of the cosmic history, thereby helping to explain the gravitational dynamics during inflationary as well as late-time accelerating regimes. Furthermore, the addition of meridional effects in stellar system causes a flow of gravitational energy due to existence of vorticity tensor in the analysis.
The paper has the following format. Section 2 deals with kinematical formulations of a comoving meridional axial symmetric geometry coupled with anisotropic matter configurations. The meridional effects in stellar system causes a flow of gravitational energy due to existence of a vorticity tensor. We present $f(R)$ dark source components and a set of dynamical equations with reflection axial degrees of freedom. In Sect. 3, we discuss a viable $f(R)$ model and use a perturbation method to develop the collapse equation. Section 4 explores instability constraints. Finally, we summarize our results in the last section.

\section{Anisotropic source and field equations}

The extended configuration of the Einstein-Hilbert action is

$S_{f(R)}=\frac{1}{2 \kappa} \int \mathrm{d}^{4} x \sqrt{-g} f(R)+S_{M}$,

where $\kappa, f(R), S_{M}, T_{\alpha \beta}$ are coupling constant, matter action, a non-linear Ricci curvature function, and the usual stress-energy tensor, respectively. The variation of the above action with respect to $g_{\alpha \beta}$ provides the field equations

$f_{R} R_{\alpha \beta}-\nabla_{\alpha} \nabla_{\beta} f_{R}-g_{\alpha \beta}\left(\frac{1}{2} f-\square f_{R}\right)=\kappa T_{\alpha \beta}$,

where $\square, \nabla_{\alpha}, f_{R}$ are the D'Alembert, the covariant derivative and $\frac{\mathrm{d} f}{\mathrm{~d} R}$ operators, respectively. Equation (2) can be written in terms of the Einstein tensor as

$G_{\alpha \beta}=\frac{\kappa}{f_{R}}\left(T_{\alpha \beta}+T_{\alpha \beta}\right)$,

where

$\stackrel{(D)}{T_{\alpha \beta}}=\frac{1}{\kappa}\left\{\frac{R}{2}\left(\frac{f}{R}-f_{R}\right) g_{\alpha \beta}-\square f_{R} g_{\alpha \beta}+\nabla_{\alpha} \nabla_{\beta} f_{R}\right\}$

is the stress-energy tensor indicating the $f(R)$ contribution in the dynamics of relativistic systems. We take axially symmetric metric characterizing reflection effects [24],

$$
\begin{aligned}
\mathrm{d} s^{2}= & -A^{2}(t, r, \theta) \mathrm{d} t^{2}+2 L(t, r, \theta) \mathrm{d} t \mathrm{~d} \theta \\
& +B^{2}(t, r, \theta)\left(\mathrm{d} r^{2}+r^{2} \mathrm{~d} \theta^{2}\right)+C^{2}(t, r, \theta) \mathrm{d} \phi^{2},
\end{aligned}
$$

with a locally anisotropic fluid configuration,

$$
\begin{aligned}
T_{\alpha \beta}= & (\mu+P) V_{\alpha} V_{\beta}+P g_{\alpha \beta} \\
& +\frac{1}{3}\left(\Pi_{I I}+2 \Pi_{I}\right)\left(K_{\alpha} K_{\beta}-\frac{1}{3} h_{\alpha \beta}\right) \\
& +\frac{1}{3}\left(\Pi_{I}+2 \Pi_{I I}\right)\left(N_{\alpha} N_{\beta}-\frac{1}{3} h_{\alpha \beta}\right) \\
& +\Pi_{K N}\left(K_{\alpha} N_{\beta}+K_{\beta} N_{\alpha}\right),
\end{aligned}
$$


where $\mu, P, \Pi_{I}, \Pi_{I I}, \Pi_{K L}$, and $h_{\alpha \beta}$ are the fluid energy density, the pressure, anisotropic scalars, and the projection tensor, respectively. The matter four velocity, $V_{\alpha}$, and spacelike vectors $S_{\alpha}, K_{\alpha}$, and $N_{\alpha}$ in comoving coordinates are

$V^{\alpha}=\frac{1}{A} \delta_{\alpha}^{0}, V_{\alpha}=-A \delta_{\alpha}^{0}+\frac{L}{A} \delta_{\alpha}^{2}, S_{\alpha}=C \delta_{\alpha}^{3}, K_{\alpha}=B \delta_{\alpha}^{1}$,

$N_{\alpha}=\frac{\sqrt{\Delta}}{A} \delta_{\alpha}^{2}$,

where $\Delta=(A B r)^{2}+L^{2}$, which obey the following constraints:

$K^{\alpha} N_{\alpha}=K^{\alpha} S_{\alpha}=S^{\alpha} N_{\alpha}=V_{\alpha} K^{\alpha}=V^{\alpha} N_{\alpha}=V^{\alpha} S_{\alpha}=0$,

$K_{\alpha} K^{\alpha}=N_{\alpha} N^{\alpha}=S_{\alpha} S^{\alpha}=-V^{\alpha} V_{\alpha}=1$.

The fluid pressure and its anisotropic scalars can be expressed alternatively in terms of the projection tensor and spacelike vectors, respectively, as

$P=\frac{1}{3} h^{\alpha \beta} T_{\alpha \beta}, \quad \Pi_{I}=\left(2 K^{\alpha} K^{\beta}-S^{\alpha} S^{\beta}-N^{\alpha} N^{\beta}\right) T_{\alpha \beta}$,

$\Pi_{K N}=K^{\alpha} N^{\beta} T_{\alpha \beta}, \Pi_{I I}=\left(2 N^{\alpha} N^{\beta}-K^{\alpha} K^{\beta}-S^{\alpha} S^{\beta}\right) T_{\alpha \beta}$.

The non-zero components of the effective stress-energy tensor (4) are

$$
T^{\alpha \beta}=\left[\begin{array}{cccc}
V_{1}+W_{1} & X_{1}+Y_{1} & X_{3}+Y_{3} & 0 \\
X_{1}+Y_{1} & V_{2}+W_{2} & X_{2}+Y_{2} & 0 \\
X_{3}+Y_{3} & X_{2}+Y_{2} & V_{3}+W_{3} & 0 \\
0 & 0 & 0 & V_{4}+W_{4}
\end{array}\right],
$$

where the dark source $f(R)$ terms $V_{i}, W_{i}$, and $X_{j}, Y_{j}$ are diagonal and non-diagonal components of the effective energy-momentum tensor (4), respectively, in which $W_{i}$ and $Y_{j}$ incorporate axial reflection effects with $f(R)$ extra degrees of freedom. By choosing $X_{j}$ and $Y_{j}$ equal to zero along with $\Delta \rightarrow A^{2} B^{2} r^{2}$, higher curvature terms of the restricted axisymmetric metric can be found. However, the inclusion of these terms along with the anisotropy in the usual stress-energy tensor ensure the propagation of gravitational radiation in the environment [40].

The kinematical quantity controlling the local spinning motion of anisotropic matter distributions is the vorticity tensor, which for a meridional axially symmetric metric can be expressed in terms of $K_{\alpha}$ and $N_{\alpha}$ as

$\Omega_{\alpha \beta}=\Omega\left(K_{\beta} N_{\alpha}-N_{\beta} K_{\alpha}\right)$,

where

$\Omega=\frac{L}{2 B \sqrt{\Delta}}\left(\frac{L^{\prime}}{L}-\frac{2 A^{\prime}}{A}\right)$ is known as the vorticity scalar. Here the prime stands for $\frac{\partial}{\partial r}$. There exists only one independent non-zero vorticity component along the $r \theta$ direction. The existence of the vorticity scalar is directly related to the existence of reflection effects of the axisymmetric spacetime, as it is controlled by the nondiagonal structure coefficient, $L$. Thus, if one takes $\Omega=0$ over the dynamical evolution of the axial anisotropic spacetime, this imparts a null value to the non-diagonal scale factor, whose dynamics has already been discussed [39].

In order to evaluate the dynamical evolution equations for an axially symmetric relativistic celestial body with $f(R)$ background, we consider

$\left|T^{\alpha \beta}+T^{(D)}\right|_{; \beta}=0$,

which yields

$$
\begin{aligned}
\dot{\mu} & -\mu\left[\frac{\dot{B}}{B}+\frac{\dot{C}}{C}+\frac{1}{\Delta}\left(r^{2} A \dot{A} B^{2}+L \dot{L}+r^{2} A^{2} B \dot{B}\right)\right] \\
& +\frac{A B^{2}(\mu+P)}{\Delta}\left[r^{2}\left(\frac{2 \dot{B}}{B}+\frac{\dot{C}}{C}\right)\right. \\
& \left.+\frac{L^{2}}{A^{2} B^{2}}\left(\frac{\dot{B}}{B}-\frac{\dot{A}}{A}+\frac{\dot{L}}{L}+\frac{\dot{C}}{C}\right)\right] \\
& +\frac{\Pi_{I}}{3 A}\left(\frac{\dot{B}}{B}-\frac{\dot{C}}{C}\right)+\frac{\Pi_{I I}}{3 \Delta}\left\{A B^{2} r^{2}\left(\frac{\dot{B}}{B}-\frac{\dot{C}}{C}\right)\right. \\
& \left.+\frac{L^{2}}{A}\left(\frac{\dot{L}}{L}-\frac{\dot{A}}{A}-\frac{\dot{C}}{C}\right)\right\} \\
& +\left(\frac{B^{2} r^{2} A \dot{A}}{\Delta}+\frac{\dot{C}}{C}\right) V_{1}+D_{0}(t, r, \theta)=0,
\end{aligned}
$$

$$
\begin{aligned}
P^{\prime} & +\frac{2}{9}\left(2 \Pi_{I}^{\prime}+\Pi_{I I}^{\prime}\right)+\left[P+\frac{2}{9}\left(2 \Pi_{I}+\Pi_{I I}\right)\right] \\
& \times\left[\frac{C^{\prime}}{C}+\frac{3 L L^{\prime}}{2 \Delta}+\frac{r^{2} A^{2} B^{2}}{\Delta}\left(\frac{A^{\prime}}{A}\right.\right. \\
& \left.\left.+\frac{2 B^{\prime}}{B}+\frac{2}{r}-\frac{(r B)^{\prime}}{r B}\right)\right]-\frac{r^{2} A B^{5}}{\Delta^{3 / 2}} \\
& \times\left[\Pi_{K N, \theta}-\left\{\frac{A_{\theta}}{A}+\frac{6 B_{\theta}}{B}+\frac{C_{\theta}}{C}+\frac{4 r^{2} A^{2} B^{2}}{\Delta}\right.\right. \\
& \left.\left.\times\left(\frac{A_{\theta}}{A}+\frac{B_{\theta}}{B}\right)+\frac{4 L L_{\theta}}{\Delta}\right\} \Pi_{K L}\right]+\frac{\mu r^{4} A^{4} B^{4}}{\Delta^{2}} \\
& \times\left(B \dot{B}+\frac{A^{\prime}}{A}-\frac{L A_{\theta}}{r^{2} A B^{2}}\right)-\left(\frac{(r B)^{\prime}}{r B}\right. \\
& \left.+\frac{L}{2 L^{\prime}}\right) \frac{\mu r^{2} A^{2} L^{2} B^{2}}{\Delta^{2}}+\left(\frac{3 \dot{B}}{B}+\frac{r^{2} B^{2} A \dot{A}}{\Delta}+\frac{\dot{C}}{C}\right) \\
& \times X_{1}+D_{1}(t, r, \theta)=0,
\end{aligned}
$$




$$
\begin{aligned}
& \frac{\mu r^{2} A^{2} B^{2} L}{\Delta^{2}}\left[\frac{\dot{\mu}}{\mu}+\frac{\dot{A}}{A}+\frac{3 \dot{B}}{B}+\frac{\dot{L}}{L}+\frac{\dot{C}}{C}+\frac{1}{r^{2} B^{2}}\right. \\
& \times\left(\frac{\mu_{\theta}}{\mu}+\frac{2 L_{\theta}}{L}+\frac{2 A_{\theta}}{A}\right)+\frac{1}{\Delta}\left\{4 r^{2} A^{2}\right. \\
& \times\left(\frac{\dot{A}}{A}+\frac{\dot{B}}{B}\right)-\frac{4 \dot{L}}{L}-L A^{2}\left(\frac{5 A_{\theta}}{A}+\frac{2 B_{\theta}}{B}\right) \\
& \left.\left.+r^{2} A^{2} B^{2}\left(\frac{\dot{L}}{L}+\frac{\dot{B}}{B}\right)+\frac{r^{2} A^{3} B^{2} A_{\theta}}{L}\right\}-\frac{4 L^{2} L_{\theta} \Delta}{r^{2} B^{2}}\right] \\
& +\frac{\mu A^{2} L^{2}}{\Delta^{2}}\left\{\frac{B_{\theta}}{B}+\frac{C_{\theta}}{C}-\frac{r^{2} B L \dot{B}}{\Delta}\right\}-\frac{r^{3} A B^{3} \Pi_{K N}}{\Delta^{\frac{3}{2}}} \\
& \times\left[\frac{\Pi_{K N}^{\prime}}{\Pi_{K N}}+\frac{3}{r}+\frac{4 B^{\prime}}{B}+\frac{A^{\prime}}{A}+\frac{C^{\prime}}{C}+\frac{3}{\Delta}\right. \\
& \left.\times\left\{\frac{L L^{\prime}}{2}+r^{2} A^{2} B^{2}\left(\frac{3}{r}+\frac{2 A^{\prime}}{A}+\frac{3 B^{\prime}}{B}\right)\right\}+\frac{7 L L^{\prime}}{2 \Delta}\right] \\
& +\frac{1}{\Delta}\left\{P+\frac{2}{9}\left(\Pi_{I}+2 \Pi_{I I}\right)\right\} \\
& \times\left[\frac { r ^ { 2 } A ^ { 2 } B ^ { 2 } } { \Delta } \left\{\left(2 A^{2}+A\right)\left(\frac{A_{\theta}}{A}+\frac{B_{\theta}}{B}\right)-\frac{L \dot{B}}{B}\right.\right. \\
& \left.+\frac{2 A B_{\theta}}{B}\right\}+2 A A_{\theta}+\frac{A^{2} C_{\theta}}{C}-\frac{r^{2} B L \dot{L}}{\Delta} \\
& \left.+\frac{2 A^{2} L L_{\theta}}{\Delta}-\frac{L \dot{B}}{B}\right]-\frac{P}{C \Delta}(L \dot{C} \\
& \left.+A^{2} C_{\theta}\right)+\frac{A^{2}}{\Delta}\left\{P_{\theta}+\frac{2}{9}\left(\Pi_{I, \theta}+2 \Pi_{I I, \theta}\right)\right\} \\
& +D_{2}(t, r, \theta)=0,
\end{aligned}
$$

where $D_{0}, D_{1}$, and $D_{2}$ are $f(R)$ corrections given in Appendix A. Here the overdot and subscript $\theta$ stand for $\frac{\partial}{\partial t}$ and $\frac{\partial}{\partial \theta}$, respectively. The second of the above equations is known as the generalized Euler equation.

\section{$3 f(R)$ model and perturbation scheme}

Many inflationary models in the early universe are established on scalar fields coming in supergravity and superstring theories. The first model of inflation was suggested by Starobinsky, which deals with a conformal anomaly in quantum gravity [41] given by [42]

$f(R)=R+\epsilon R^{n}$,

where $n$ can be positive or negative integer. This model explains the present universe's acceleration due to the presence of dark energy and can serve as power-law inflation, i.e. an exponential expansion and ordinary inflation incorporating a minimally coupled scalar field. Here $\epsilon \sim \frac{1}{M^{2 n-2}}>0$ for $n>0$, and $M$ has mass dimensions. Since $f(R)$ gravity can be used as an alternative for dark matter [43-46] in addition to dark energy at cluster as well as stellar scales, this model with $n=2$ was claimed both as a dark matter model with $\epsilon=\frac{1}{6 M^{2}}$ [47-49] and as a dark energy model. The value of $M$ is chosen to be $2.7 \times 10^{-12} \mathrm{GeV}$ along with $\epsilon \leq 2.3 \times 10^{22} \mathrm{Ge} / \mathrm{V}^{2}$ for dark matter cosmology [50]. All GR solutions can be found by taking the limit $f(R) \rightarrow R$.

Here, we use perturbation method [26-29] to explore a modified collapse equation for a meridional axially symmetric anisotropic geometry. For very small values of the perturbation parameter $\alpha$ with $0<\alpha \ll 1$, we take effects up to $O(\alpha)$. We first suppose that the system is in hydrostatic equilibrium at $t=0$, however, on departing from this state, the system depends upon the same time dependence factor $T(t)$ on all its structure coefficients. The structure and matter variables can be perturbed as follows:

$\mathcal{S}(t, r, \theta)=\mathcal{S}_{0}(r, \theta)+\alpha T(t) s(r, \theta)$,

$\mathcal{M}(t, r, \theta)=\mathcal{M}_{0}(r, \theta)+\alpha \bar{m}(t, r, \theta)$,

where $\mathcal{S}$ represents a perturbation method applicable to the structural coefficients of Eq. (5), i.e., $A, B, C, L$, and to the Ricci scalar, $R$, which after perturbation denotes $s$ as $a, b, c, l$, and $e$, respectively. Equation (15) indicates the perturbation method of the matter variables [these matter variables are taken from Eq. (6)]. Thus the allocation of $\mathcal{M}$ will be $\mu, P, \Pi_{a}, a=1,2,3$, and the corresponding perturbed quantities will be represented by placing a bar over that. However, the perturbation technique for the $f(R)$ model is given as follows:

$$
\begin{aligned}
& \left.f(t, r)=\left[R_{0}(r)+\epsilon R_{0}^{n}\right)\right]+\alpha T(t) e(r)\left[1-\epsilon n R_{0}^{n-1}\right], \\
& f_{R}(t, r)=1+\epsilon n R_{0}^{n-1}+\alpha T(t) e(r) n \epsilon(n-1) R_{0}^{n-2},
\end{aligned}
$$

where $R_{0}$ represents the static distribution of the Ricci scalar. Using Eqs. (14)-(17), the first of the dynamical equations is satisfied trivially, while the rest of the dynamical equations (11) and (12) at $t=0$ give

$$
\begin{aligned}
P_{0}^{\prime} & +\frac{2}{9}\left(2 \Pi_{I 0}^{\prime}+\Pi_{I I 0}^{\prime}\right)+\left[P_{0}+\frac{2}{9}\left(2 \Pi_{I 0}+\Pi_{I I 0}\right)\right] \\
& \times\left[\frac{C_{0}^{\prime}}{C_{0}}+\frac{3 L_{0} L_{0}^{\prime}}{2 \Delta_{0}}+\frac{r^{2} A_{0}^{2} B_{0}^{2}}{\Delta_{0}} \times\left(\frac{A_{0}^{\prime}}{A_{0}}+\frac{1}{r}\right)\right] \\
& -\frac{r^{3} A_{0} B_{0}^{5}}{\Delta_{0}^{\frac{3}{2}}} \Pi_{K N 0, \theta}-\frac{r^{3} A_{0} B_{0}^{5}}{\Delta_{0}^{\frac{3}{2}}}\left\{\frac{A_{0 \theta}}{A}+\frac{6 B_{0 \theta}}{B_{0}}+\frac{C_{0 \theta}}{C_{0}}\right. \\
& \left.+\frac{4 L_{0} L_{0 \theta}}{\Delta_{0}}+\frac{4 r^{2} A_{0}^{2} B_{0}^{2}}{\Delta_{0}}\left(\frac{A_{0 \theta}}{A_{0}}+\frac{B_{0 \theta}}{B_{0}}\right)\right\} \\
& +\frac{\mu r^{4} A_{0}^{4} B_{0}^{4}}{\Delta_{0}^{2}}\left(\frac{A_{0}^{\prime}}{A_{0}}-\frac{L_{0} A_{0 \theta}}{r^{2} A_{0} B_{0}^{2}}\right)-\frac{\mu_{0} r^{2} A_{0}^{2} L_{0}^{2} B_{0}^{2}}{\Delta_{0}^{2}} \\
& \times\left(\frac{L_{0}}{2 L_{0}^{\prime}}+\frac{1}{r}+\frac{B_{0}^{\prime}}{B_{0}}\right)+D_{1 S}=0,
\end{aligned}
$$




$$
\begin{aligned}
& \frac{\mu_{0} r^{2} A_{0}^{2} B_{0}^{2} L_{0}}{\Delta^{2}}\left[+\frac{1}{r^{2} B_{0}^{2}}\left(\frac{\mu_{0 \theta}}{\mu_{0}}+\frac{2 L_{0 \theta}}{L_{0}}+\frac{2 A_{0 \theta}}{A_{0}}\right)\right. \\
& -\frac{1}{\Delta_{0}}\left\{L_{0} A_{0}^{2}\left(\frac{5 A_{0 \theta}}{A_{0}}+\frac{2 B_{0 \theta}}{B_{0}}\right)-\frac{r^{2} A_{0}^{3} B_{0}^{2} A_{0 \theta}}{L_{0}}\right\} \\
& \left.-\frac{4 L_{0}^{2} L_{0 \theta} \Delta_{0}}{r^{2} B_{0}^{2}}\right]+\frac{\mu A_{0}^{2} L_{0}^{2}}{\Delta_{0}^{2}}\left\{\frac{B_{0 \theta}}{B_{0}}+\frac{C_{\theta 0}}{C}\right\} \\
& -\frac{r^{3} A_{0} B_{0}^{3} \Pi_{K N 0}}{\Delta_{0}^{3 / 2}}\left[\frac{3}{r}+\frac{\Pi_{K N 0}^{\prime}}{\Pi_{K N 0}}+\frac{4 B_{0}^{\prime}}{B_{0}}\right. \\
& +\frac{A_{0}^{\prime}}{A_{0}}+\frac{C_{0}^{\prime}}{C_{0}}+\frac{3}{\Delta_{0}} \\
& \times\left\{\frac{L_{0} L_{0}^{\prime}}{2}+r^{2} A_{0}^{2} B_{0}^{2}\left(\frac{3}{r}+\frac{2 A_{0}^{\prime}}{A_{0}}+\frac{3 B_{0}^{\prime}}{B_{0}}\right)\right\} \\
& \left.+\frac{7 L_{0} L_{0}^{\prime}}{2 r \Delta_{0}}\right]+\frac{1}{\Delta_{0}}\left\{P_{0}+\frac{2}{9}\left(\Pi_{I 0}+2 \Pi_{I I 0}\right)\right\} \\
& + \\
& +
\end{aligned}
$$

The static $f(R)$ contribution of the second and third conservation equations are denoted by $D_{1 \mathrm{~S}}$ and $D_{2 \mathrm{~S}}$, respectively, and they can be calculated very easily from Eqs. (A.2) and (A.3) after using the perturbation method. Using Eqs. (14)-(17), the non-static perturbed axial dynamical equation (10) will take the form

$$
\begin{aligned}
\dot{\bar{\mu}} & +\left[\mu_{0}\left\{\frac{b}{B_{0}}+\frac{c}{C_{0}}+\frac{1}{\Delta_{0}}\left(r^{2} a A_{0}^{2} B_{0}^{2}+l L_{0}+r^{2} b B_{0} L_{0}\right)\right\}\right. \\
& +\left(\mu_{0}+P_{0}\right) \frac{A_{0}^{2} B_{0}^{2}}{\Delta_{0}^{2}} \\
& \times\left\{r^{2}\left(\frac{2 b}{B_{0}}+\frac{2 c}{C_{0}}\right)+\frac{L_{0}^{2}}{A_{0}^{2} B_{0}^{2}}\left(\frac{b}{B_{0}}+\frac{l}{L_{0}}-\frac{a}{A_{0}}+\frac{c}{C_{0}}\right)\right\} \\
& +\frac{\Pi_{I 0}}{3}\left(\frac{b}{B_{0}}-\frac{c}{C_{0}}\right) \\
& +\frac{\Pi_{I I 0}}{3 \Delta_{0}}\left\{r^{2} A_{0}^{2} B_{0}^{2}\left(\frac{b}{B_{0}}-\frac{c}{C_{0}}\right)+L_{0}^{2}\left(\frac{l}{L_{0}}-\frac{a}{A_{0}}-\frac{c}{C_{0}}\right)\right\} \\
& \left.+D_{3}(r, \theta)\right] \dot{T}=0,
\end{aligned}
$$

where $D_{3}$ represents $f(R)$ corrections which can be obtained from the expressions $g(t, r, \theta)$ and $h(t, r, \theta)$ given in Appendix A. Substituting Eq. (13) in Eq. (8) and then employing the perturbation method, one can obtain the $f(R)$ dynamical quantities, $V_{i}, W_{i}, X_{j}, Y_{j}$ whose values upon substitution in Eqs. (A.4) and (A.5) yield $D_{3}$ such that $g(t, r, \theta)+h(t, r, \theta)=D_{3} \dot{T}$. Integration of the above equation gives

$\bar{\mu}=-\chi(r, \theta) T$,

where

$$
\begin{aligned}
\chi= & {\left[\mu_{0}\left\{\frac{b}{B_{0}}+\frac{c}{C_{0}}+\frac{1}{\Delta_{0}}\left(r^{2} a A_{0}^{2} B_{0}^{2}+l L_{0}+r^{2} b B_{0} L_{0}\right)\right\}\right.} \\
& +\left(\mu_{0}+P_{0}\right) \frac{A_{0}^{2} B_{0}^{2}}{\Delta_{0}^{2}}\left\{r^{2}\left(\frac{2 b}{B_{0}}+\frac{2 c}{C_{0}}\right)\right. \\
& \left.+\frac{L_{0}^{2}}{A_{0}^{2} B_{0}^{2}}\left(\frac{b}{B_{0}}+\frac{l}{L_{0}}-\frac{a}{A_{0}}+\frac{c}{C_{0}}\right)\right\} \\
& +\frac{\Pi_{I 0}}{3}\left(\frac{b}{B_{0}}-\frac{c}{C_{0}}\right)+\frac{\Pi_{I I 0}}{3 \Delta_{0}}\left\{r^{2} A_{0}^{2} B_{0}^{2}\left(\frac{b}{B_{0}}-\frac{c}{C_{0}}\right)\right. \\
& \left.\left.+L_{0}^{2}\left(\frac{l}{L_{0}}-\frac{a}{A_{0}}-\frac{c}{C_{0}}\right)\right\}+D_{3}(r, \theta)\right] .
\end{aligned}
$$

Now, we evaluate the $t \theta$ component of the metric $f(R)$ field equations (3) and then using the perturbation scheme along with some manipulations, it follows that

$\varrho_{1} \ddot{T}+\varrho_{2} \dot{T}+\varrho_{3} T=0$,

where the quantities $\varrho_{i}$ contain combinations of the meridional axial geometric functions as well as $R+\epsilon R^{n}$ corrections, depending upon the $r$ and $\theta$ coordinates, and they are assumed to be positive. More specifically, these quantities incorporate non-perturbed as well as perturbed terms. There exist oscillating as well as non-oscillating solutions of the above equation, which represent unstable as well as stable models of evolving relativistic stellar systems, respectively. We confine ourselves to obtaining solutions for a collapsing relativistic system. Thus we limit our perturbation parameters, $a, b, c, e$, and $l$ to be positive definite quantities for which we obtain $\omega^{2}>0$. In this context, the solution of Eq. (21) is given by

$T(t)=-\exp (\omega t), \quad$ where $\omega^{2}=\frac{-\varrho_{2}+\sqrt{\varrho_{2}^{2}-4 \varrho_{1} \varrho_{3}}}{2 \varrho_{1}}$.

Using the perturbation technique, the non-static distributions of Eq. (11), after using Eq. (22), are written as

$$
\begin{aligned}
& \frac{1}{B_{0}^{2}}\left\{\bar{P}^{\prime}+\frac{2}{9}\left(2 \bar{\Pi}_{I}^{\prime}+\bar{\Pi}_{I I}^{\prime}\right)\right\}+\frac{1}{B_{0}^{2}}\left\{\bar{P}+\frac{2}{9}\left(2 \bar{\Pi}_{I}+\bar{\Pi}_{I I}\right)\right\} \\
& \quad \times\left\{\frac{C_{0}^{\prime}}{C_{0}}+\frac{3 L_{0} L_{0}^{\prime}}{2 \Delta_{0}}+\left(\frac{1}{r}+\frac{A_{0}^{\prime}}{A_{0}}\right) \frac{r^{2} A_{0}^{2} B_{0}^{2}}{\Delta_{0}}\right\}
\end{aligned}
$$




$$
\begin{aligned}
& -\frac{r^{3} A_{0} B_{0}^{3}}{\Delta_{0}^{3 / 2}} \bar{\Pi}_{K N, \theta}-\bar{\Pi}_{K N} \frac{r^{3} A_{0} B_{0}^{3}}{\Delta_{0}^{3 / 2}}\left\{\frac{A_{0 \theta}}{A_{0}}+\frac{6 B_{0 \theta}}{B_{0}}\right. \\
& \left.+\frac{4 L_{0} L_{0} \theta}{\Delta_{0}}+\frac{C_{0 \theta}}{C_{0}}+\frac{4 r^{2} A_{0} B_{0}^{2}}{\Delta_{0}}\left(\frac{A_{0 \theta}}{A_{0}}+\frac{B_{0 \theta}}{B_{0}}\right)\right\} \\
& +\frac{\bar{\mu} r^{4} A_{0}^{4}}{\Delta_{0}^{2}}\left(\frac{A_{0}^{\prime}}{A_{0}}-\frac{L_{0} A_{0 \theta}}{r^{2} A_{0} B_{0}^{2}}\right)-\bar{\mu} L_{0}^{2} r^{2} \frac{A_{0}^{2}}{\Delta_{0}^{2}} \\
& \times\left(\frac{L_{0}^{\prime}}{2 L_{0}}+\frac{B_{0}^{\prime}}{B_{0}}\right)-\frac{2 b}{B_{0}^{3}}\left\{P_{0}^{\prime}+\frac{2}{9}\left(2 \Pi_{I 0}^{\prime}+\Pi_{I I 0}^{\prime}\right)\right\} \\
& \times T+\frac{r^{3} A_{0} B_{0}^{3}}{\Delta_{0}^{3 / 2}} \Pi_{K N 0, \theta}\left(\frac{a}{A_{0}}+\frac{3 b}{B_{0}}-\frac{3 d}{\Delta_{0}}\right) T \\
& +\frac{l L_{0} X_{10}}{\Delta_{0}}+\frac{r^{2} A_{0}^{2} b B_{0}}{\Delta_{0}} X_{30}+[\Upsilon+\Phi+\zeta] T=0,
\end{aligned}
$$

where $\Upsilon, \zeta$, and $\Phi$ are mentioned in Appendix A. The quantity controlling the reflection degrees of freedom along with the $f(R)$ corrections of an axisymmetric celestial body is $\Upsilon$. However, the expression $\Phi$ incorporates a gravitational contribution due to $f(R)$ gravity, while $\zeta$ is the remaining part of the non-static perturbed generalized Euler equation holding the usual Einstein gravity effects.

In view of the second law of thermodynamics, we can link perturbed anisotropic quantities with the energy density by an equation of state as [51]

$\bar{P}_{i}=\Gamma_{1} \frac{P_{i 0}}{\mu_{0}+P_{i 0}} \bar{\mu}$,

where $\Gamma_{1}$ is a fluid stiffness parameter, also known as the adiabatic index. This measures pressure variations of the matter configurations with respect to energy density. In our analysis, $\Gamma_{1}$ will be treated as a constant identity. Using Eqs. (20) and (24), we have

$$
\begin{aligned}
& \bar{\Pi}_{K N}=-\Gamma_{1} \frac{\Pi_{K N 0}}{\mu_{0}+\Pi_{K N 0}} \chi T, \quad \bar{P}=-\Gamma_{1} \frac{P_{0}}{\mu_{0}+P_{0}} \chi T, \\
& \bar{\Pi}_{I}=-\Gamma_{1} \frac{\Pi_{I 0}}{\mu_{0}+\Pi_{I 0}} \chi T, \quad \bar{\Pi}_{I I}=-\Gamma_{1} \frac{\Pi_{I I 0}}{\mu_{0}+\Pi_{I I 0}} \chi T .
\end{aligned}
$$

Using Eq. (20) as well as the above relations in Eq. (23), we obtain

$$
\begin{aligned}
& -\frac{1}{B_{0}^{2}} \Gamma_{1} \phi^{\prime} T-\frac{1}{B_{0}^{2}} \Gamma_{1} \phi T \\
& \times\left\{\frac{C_{0}^{\prime}}{C_{0}}+\frac{3 L_{0} L_{0}^{\prime}}{2 \Delta_{0}}+\left(\frac{1}{r}+\frac{A_{0}^{\prime}}{A_{0}}\right) \frac{r^{2} A_{0}^{2} B_{0}^{2}}{\Delta_{0}}\right\} \\
& -\frac{r^{3} A_{0} B_{0}^{3}}{\Delta_{0}^{3 / 2}} \Gamma_{1} T\left(\frac{\Pi_{K N 0} \chi}{\mu_{0}+\Pi_{K N 0}}\right)_{\theta}-\frac{\Pi_{K N 0 \chi}}{\mu_{0}+\Pi_{K N 0}} \frac{r^{3} A_{0} B_{0}^{3}}{\Delta_{0}^{3 / 2}} \\
& \quad \times\left\{\frac{A_{0 \theta}}{A_{0}}+\frac{4 r^{2} A_{0} B_{0}^{2}}{\Delta_{0}}\left(\frac{A_{0 \theta}}{A_{0}}+\frac{B_{0 \theta}}{B_{0}}\right)\right.
\end{aligned}
$$

$$
\begin{aligned}
& \left.+\frac{C_{0 \theta}}{C_{0}}+\frac{6 B_{0 \theta}}{B_{0}}+\frac{4 L_{0} L_{0} \theta}{\Delta_{0}}\right\} \\
& -T \frac{\chi r^{4} A_{0}^{4}}{\Delta_{0}^{2}}\left(\frac{A_{0}^{\prime}}{A_{0}}-\frac{L_{0} A_{0 \theta}}{r^{2} A_{0} B_{0}^{2}}\right)+T \chi L_{0}^{2} r^{2} \frac{A_{0}^{2}}{\Delta_{0}^{2}}\left(\frac{L_{0}^{\prime}}{2 L_{0}}\right. \\
& \left.+\frac{B_{0}^{\prime}}{B_{0}}\right)-\frac{2 b}{B_{0}^{3}}\left\{P_{0}^{\prime}+\frac{2}{9}\left(2 \Pi_{I 0}^{\prime}+\Pi_{I I 0}^{\prime}\right)\right\} \\
& \times T+\frac{r^{3} A_{0} B_{0}^{3}}{\Delta_{0}^{3 / 2}}\left(\frac{a}{A_{0}}+\frac{3 b}{B_{0}}-\frac{3 d}{\Delta_{0}}\right) \\
& \times \Pi_{K N 0, \theta} T+\frac{l L_{0} X_{10}}{\Delta_{0}}+\frac{r^{2} A_{0}^{2} b B_{0}}{\Delta_{0}} X_{30} \\
& +[\Upsilon+\Phi+\zeta] T=0,
\end{aligned}
$$

where $\phi=\frac{P_{0} \chi}{\left(\mu_{0}+P_{0}\right)}+\frac{4 \Pi_{I 0 \chi}}{9\left(\mu_{0}+\Pi_{I 0}\right)}+\frac{2 \Pi_{I I 0 \chi}}{9\left(\mu_{0}+\Pi_{I I 0}\right)}$. The above equation is known as the collapse equation of axisymmetric stellar objects characterizing the meridional and $f(R)$ extraorder degrees of freedom.

\section{Instability regions}

Now we proceed to calculate constraints at which meridional axial symmetric stellar systems undergo an instability window at both the $\mathrm{N}$ and the $\mathrm{pN}$ eras with an $f(R)$ background. We also examine the role of the stiffness parameter $\Gamma_{1}$ in this scenario. We reduce our results to previously found limiting cases. The formulation of the instability constraints should be compatible with the Tolman-Oppenheimer-Volkoff (TOV) equation. Such a type of equation constrains the relativistic stellar structure coupled with the matter distribution at the phase of static gravitational equilibrium. In this respect, Barausse et al. [52] investigated hydrostatic equilibrium phases of relativistic models by obtaining a modified version of the TOV equation in $f(R)$ gravity. Recently, Astashenok et al. [53] calculated an extended version of the TOV equation with an equation of state in the realm of cubic as well as quadratic corrections and found that such an equation can be used to describe viable models of compact objects. Here, we formulate the TOV equation that will help us to obtain some limits on the fluid energy density and its derivatives to avoid a curvature divergence at the stellar boundary. The 11 and 22 components of the metric $f(R)$ field equations, respectively, provide us with

$$
\begin{aligned}
\frac{A^{\prime}}{A}= & \frac{B^{2}}{\gamma}\left[\frac{\kappa}{f_{R}}\left(P+\frac{\Pi_{I}}{3}+\frac{\xi_{2}}{\kappa B^{2}}\right)-\frac{\xi_{1}}{\Delta^{2} B^{2}}\right], \\
\frac{A_{\theta}}{A}= & \frac{1}{\gamma_{1}}\left[\frac { \kappa } { f _ { R } } \left\{\frac{\mu L^{2}}{A^{2}}+\frac{\Delta}{A^{2}}\left(P+\frac{2}{9}\left(\Pi_{I I}+2 \Pi_{I}\right)\right)\right.\right. \\
& \left.\left.+\frac{\xi_{4}}{\kappa}\right\}-\frac{\xi_{3}}{4 A^{2}}\right],
\end{aligned}
$$


where

$$
\begin{aligned}
& \gamma=\frac{A^{2} B^{2} r^{2}}{\Delta}\left[\frac{16}{\Delta}\left(1+\frac{r C^{\prime}}{C}+\frac{r B^{\prime}}{B}\right)+\frac{r f_{R}^{\prime}}{f_{R}}\right], \\
& \gamma_{1}=\frac{A^{2} B^{2} r^{2} L^{2}}{\Delta^{2}}\left(\frac{r^{2} B^{2} \dot{C}}{L C}-\frac{2 B_{\theta}}{B} 2 \frac{2 C_{\theta}}{C}\right) \\
& -\frac{r^{2} B^{2} A^{2} f_{R \theta}}{\kappa f_{R}}\left(\frac{r^{2} A^{2} B^{2}}{\Delta}-\frac{L^{2}}{\Delta}\right) \\
& -\frac{B^{4} A^{4} r^{4}}{\Delta^{2}}\left(\frac{C_{\theta}}{C}+\frac{B_{\theta}}{B}\right) \text {, } \\
& \xi_{1}=\Delta^{2} G_{11}-4 \Delta r^{2} A^{2} b^{2} \frac{A^{\prime}}{A}\left(\frac{4}{r}+\frac{4 C^{\prime}}{C}+\frac{4 B^{\prime}}{B}\right), \\
& \xi_{2}=T_{11}^{(D)}+\frac{A^{2} B^{2} r^{2} f_{R}^{\prime} A^{\prime}}{\kappa A \Delta}, \\
& \xi_{3}=4 \Delta^{2} G_{22}+4 B^{4} A^{4} r^{4}\left(\frac{A_{\theta} C_{\theta}}{A C}+\frac{A_{\theta} B_{\theta}}{A B}\right) \\
& -4 A^{2} B^{2} r^{2} L^{2}\left(\frac{r^{2} B^{2} \dot{C} A_{\theta}}{L C A}-\frac{2 A_{\theta} B_{\theta}}{A B}-\frac{2 A_{\theta} C_{\theta}}{A C}\right) \text {, } \\
& \xi_{4}=T_{22}-\frac{A^{2} B^{2} r^{2} f_{R \theta}}{\Delta}\left(\frac{r^{2} B^{2} A A_{\theta}}{\Delta}-\frac{L^{2} A_{\theta}}{A \Delta}\right) \text {. }
\end{aligned}
$$

The corresponding Misner-Sharp mass function [54] takes the form

$m_{\mathrm{tot}}=\frac{r^{3} B}{2}\left(\frac{r^{2} B^{2} \dot{B}^{2}}{\Delta}-\frac{B^{\prime}}{r B^{2}}-\frac{2 B^{\prime}}{r B}-\frac{A^{2} B_{\theta}^{2}}{\Delta}-\frac{2 L B_{\theta} \dot{B}}{\Delta}\right)$.

Using Eq. (30), and the second and the third laws of the conservation of the usual energy-momentum tensor as well as Eqs. (26) and (27), we obtain the TOV equations,

$$
\begin{aligned}
- & P^{\prime}=\left[\frac{9 \mu r^{4} A^{4} \psi_{m}^{4}-9 r^{2} A^{2} \psi_{m}^{2} \Delta\left\{9 P+2\left(\Pi_{I I}+2 \Pi_{I}\right)\right\}}{9 \Delta^{2}}\right] \\
& \times \frac{\psi_{m}^{2}}{\gamma}\left[\frac{\kappa}{f_{R}}\left(P+\frac{\Pi_{I}}{3}+\frac{\xi_{2}}{\kappa \psi_{m}^{2}}\right)-\frac{\xi_{1}}{\Delta^{2} \psi_{m}^{2}}\right] \\
& +\left[\xi_{5}+\frac{2}{9}\left(2 \Pi_{I I}+\Pi_{I}\right)\right], \\
& -P_{\theta}=\left[\frac{-5 \mu r^{2} A^{4} \psi_{m}^{2} L-r^{2} A^{2} \psi_{m}^{2}\left\{9 P+2\left(\Pi_{I I}+2 \Pi_{I}\right)\right\}}{9 \Delta^{2}}\right] \\
& \times \frac{\Delta}{\gamma_{1} A^{2}}\left[\frac{\kappa}{f_{R}}\left\{\frac{\mu L^{2}}{A^{2}}+\frac{\Delta}{A^{2}}\left(P+\frac{2}{9}\left(\Pi_{I I}+2 \Pi_{I}\right)\right)+\frac{\xi_{4}}{\kappa}\right\}\right. \\
& \left.+\frac{\xi_{1}}{4 A^{2}}\right]+\frac{\xi_{7} \Delta}{A^{2}} .
\end{aligned}
$$

where

$$
\begin{aligned}
\xi_{5}= & T_{; \beta}^{0 \beta}-\left(P+\frac{2}{9}\left(2 \Pi_{I I}+\Pi_{I}\right)\right) \frac{r^{2} A^{2} B^{2} A^{\prime}}{\Delta A} \\
& +\frac{A^{\prime} \mu r^{4} A^{4} B^{4}}{\Delta^{2} A}-\left(P+\frac{2}{9}\left(\Pi_{I I}+2 \Pi_{I}\right)\right)^{\prime}, \\
\xi_{6}= & \xi_{2}+\frac{A^{2} r^{2} B^{2} f_{R}^{\prime}}{\Delta}\left(\frac{B^{\prime}}{B}+\frac{1}{r}\right)-\frac{C^{\prime} f_{R}^{\prime}}{C}-\frac{L \Delta L^{\prime} f_{R}^{\prime}}{2}, \\
\xi_{7}= & T_{; \beta}^{1 \beta}-\frac{A^{2}}{\Delta} P_{\theta}+\frac{A_{\theta}}{A} \\
& \times\left[\frac{5 r^{2} A^{4} B^{2} L \mu}{\Delta^{2}}-\frac{r^{2} A^{2} B^{2}}{\Delta^{2}}\left(P+\frac{2}{9}\left(\Pi_{I I}+2 \Pi_{I}\right)\right)\right], \\
\psi_{m}= & \frac{\left(2 m_{t o t}-r^{2} B^{\prime}\right)+D \sqrt{\left(r^{2} B^{\prime}-2 m_{t o t}\right)^{2}+4 r^{4} \dot{B}^{2}\left(r A^{2} B^{\prime}-A^{2} B_{\theta}^{2}-L B_{\theta} \dot{B}\right)}}{2 \dot{B}^{2} r^{3}},
\end{aligned}
$$

where $\psi_{m}$ is calculated by making the assumption that reflection effects are far smaller than those produced by other scale factors in the evolution of an axisymmetric system. In order to examine the contributions of $f_{R}^{\prime}$ and $f_{R \theta}$ across the meridional non-static axial relativistic object, we multiply both sides of the above equations with $\frac{\mathrm{d} f}{\mathrm{~d} P}$. After some manipulations, this yields a couple of quadratic equations in $f_{R}^{\prime}$ and $f_{R \theta}$, whose solutions become

$$
\begin{aligned}
f_{R}^{\prime}= & \frac{1}{18 f_{R} C r^{3} A^{2} \psi_{m}^{2} \Delta} \\
& \times\left[-\psi_{m} r^{2} A^{2}\left(144\left(f_{R} \psi_{m} C+f_{R} \psi_{m} r C^{\prime}+C r \psi_{m}^{\prime}\right)\right.\right. \\
& \left.\left.+2 \mathcal{C}_{1} \Delta r C \psi_{m}+9 \mathcal{C}_{2} r C \psi_{m} \Delta\right)+D \sqrt{\Delta_{1}}\right] \\
f_{R \theta}= & \frac{1}{72 A^{4} B^{3} C L \Delta\left(A^{2} b^{2} r^{2}-L^{2}\right)} \\
& \times\left[-36 A^{4} f_{R} B^{2} r^{2} L^{2} \kappa\left(r^{2} B^{3} \dot{C}-2 C L b_{\theta}\right.\right. \\
& \left.-2 L B C_{\theta}\right)+36 A^{6} B^{4} L r^{4} f_{R} \kappa\left(B C_{\theta}-C B_{\theta}\right) \\
& \left.-36 A^{2} r^{2} B^{2} L C \Delta^{2}\left(r^{2} B^{2} A^{2}-L^{2}\right)+D \sqrt{\Delta_{2}}\right]
\end{aligned}
$$

where $\Delta_{1}$ and $\Delta_{2}$ are the discriminants of the $f_{R}^{\prime}$ and $f_{R \theta}$ quadratic equations, $\mathcal{C}=\left[\frac{9 \mu r^{4} A^{4} \psi_{m}^{4}-9 r^{2} A^{2} \psi_{m}^{2} \Delta\left\{9 P+2\left(\Pi_{I I}+2 \Pi_{I}\right)\right\}}{9 \Delta^{2}}\right]$ $\frac{\mathrm{d} f_{R}}{\mathrm{~d} P}, \mathcal{C}_{1}=2\left(\Pi_{I I}+2 \Pi_{I}\right)^{\prime} \frac{\mathrm{d} f_{R}}{\mathrm{~d} P}$, while $D= \pm 1$. We shall take $A_{0}=1-\varphi, B_{0}=1+\varphi$ with $\varphi=\frac{m_{0}}{r}$ for the $\mathrm{pN}$ epochs; therefore,

$$
\frac{A_{0}^{\prime}}{A_{0}}=(1+\varphi)^{\prime}(1-\varphi), \quad \frac{A_{0 \theta}}{A_{0}}=(1+\varphi)_{\theta}(1-\varphi) .
$$

Over the surface of an axial reflection relativistic star object, Eqs. (31) and (32) yield

$\frac{f_{R}^{\prime}}{f_{R}}=\frac{W_{1}(D-1)}{18 C \psi_{m} \Delta}, \quad \frac{f_{R \theta}}{f_{R}}=\frac{W_{2}(D-1)}{2}$, 
where $W_{1}=144\left(\psi_{m} C+\psi_{m} r C^{\prime}+r C \psi_{m}^{\prime}\right)$ and $W_{2}=$ $\frac{A^{2} r^{2} L \kappa}{\left(A^{2} B^{2} r^{2}-L^{2}\right)}\left(r^{2} \dot{C} \psi_{m}+2 L C \psi_{m \theta}-2 L C_{\theta} \psi_{m}\right)-A^{4} B^{2} r^{4} \kappa$ $\left(B C_{\theta}-C B_{\theta}\right)+r^{2} C \Delta^{2}\left(r^{2} A^{2} B^{2}-L^{2}\right)$. It can found from Eq. (33) that on setting $D=-1$, one can get specific forms of $\gamma$ and $\gamma_{1}$ from Eqs. (28) and (29), which will make $\frac{A^{\prime}}{A}$ and $\frac{A_{\theta}}{A}$ approach $\infty$ with $(r, \theta) \rightarrow\left(r^{-}, \theta^{-}\right)$, while a finite value of $\frac{A^{\prime}}{A}$ and $\frac{A_{\theta}}{A}$ can be achieved for $(r, \theta) \rightarrow\left(r^{+}, \theta^{+}\right)$. For a physically viable stellar model, we take $D=1$, which yields $f_{R \theta}=0=f_{R}^{\prime}$ for $(r, \theta) \rightarrow\left(r^{-}, \theta^{-}\right)$. This reinforces the continuity of $f_{R \theta}, f_{R}^{\prime}$ as well as $A^{\prime}$ over the surface of the axial stellar structure with reflection degrees of freedom.

\subsection{Newtonian approximation}

In order to evaluate the instability conditions at the $\mathrm{N}$ regime, we take $A_{0}=B_{0}=1$ and we assume the anisotropic pressure to be less than zero, which is the criterion for a collapsing celestial body. We also take configurations of initial perturbed structural coefficients to be $C_{0}=L_{0}=r$. Consequently, the collapse equation (25) turns out to be

$$
\begin{aligned}
\Gamma_{1} \phi_{\mathcal{N}}^{\prime} & +\frac{9}{4 r} \phi_{\mathcal{N}} \Gamma_{1}-\Gamma_{1} \frac{\Pi_{K N 0, \theta}}{2 r \sqrt{2}}\left(\frac{2 c}{r}+3 b+\frac{l}{r}\right)_{\theta} \\
= & \frac{3}{8 r}\left(\frac{2 c}{r}+3 b+\frac{l}{r}\right)-2 b\left[P_{0}^{\prime}\right. \\
& \left.+\frac{2}{9}\left(2 \Pi_{I 0}^{\prime}+\Pi_{I I 0}^{\prime}\right)\right]+\frac{1}{2 r \sqrt{2}}\left(2 b-\frac{l}{r}\right) \\
& +\frac{b}{2} X_{30 \mathcal{N}}+\frac{l}{2 r} X_{10 \mathcal{N}}+\Upsilon+\Phi+\zeta,
\end{aligned}
$$

where the subscript $\mathcal{N}$ indicates the evaluation of the term at the $\mathrm{N}$ regime. We assume that all terms on both sides of the above equation are positive. The instability constraint for meridional axisymmetric fluid configurations is given by

$\Gamma_{1}<\frac{\frac{3}{8 r}\left(\frac{2 c}{r}+3 b+\frac{l}{r}\right)-2 b\left[P_{0}^{\prime}+\frac{2}{9}\left(2 \Pi_{I 0}^{\prime}+\Pi_{I I 0}^{\prime}\right)\right]+\phi_{1}+\zeta_{N}}{\phi_{\mathcal{N}}^{\prime}+\frac{9}{4 r} \phi_{\mathcal{N}}-\frac{\Pi_{K N 0 . \theta}}{2 r \sqrt{2}}\left(\frac{2 c}{r}+3 b+\frac{l}{r}\right)_{\theta}}$,

where $\phi_{1}=\frac{b}{2} X_{30 \mathcal{N}}+\frac{l}{2 r} X_{10 \mathcal{N}}+\frac{1}{2 r \sqrt{2}}\left(2 b-\frac{l}{r}\right)+\Upsilon_{\mathcal{N}}+$ $\Phi_{\mathcal{N}}+\zeta_{\mathcal{N}}$. The system would be in complete hydrostatic equilibrium, if (during evolution) it can take a value equal to the right hand side of the above expression. However, on satisfying the above inequality, the system will move into the unstable phase. This constraint is being acknowledged through the $\Gamma_{1}$ parameter, thereby emphasizing the importance of the matter stiffness factor in our investigation.

\subsection{Post-Newtonian approximation}

Here, we take the axial structural coefficients for the $\mathrm{pN}$ eras and consider our outcomes up to $O(\varphi)$. Using these relations in Eq. (25), one may have the modified collapse equation at the $\mathrm{pN}$ limit. This leads toan instability inequality through the stiffness parameter

$\Gamma_{1}<\frac{r^{2}(1-4 \phi)\left\{\varphi^{\prime}+\frac{1}{r}(1-\varphi)(1-\varphi)_{\theta}\right\} \chi_{p N}+(1-2 \varphi) \frac{\chi_{p N}}{4}\left(\varphi^{\prime}+\frac{3}{2 r}\right)+\zeta_{1}}{(1-2 \varphi) \phi_{p N}^{\prime}+(1-2 \varphi) \phi_{p N}\left[\frac{7}{4 r}+\frac{1}{2}\left(\frac{1}{r}-\varphi^{\prime}\right)\right]+\zeta_{2}}$,

where

$$
\begin{aligned}
\zeta_{1}= & \Pi_{K N 0 \theta}-2 b(1-3 \varphi)\left[P_{0}^{\prime}+\frac{2}{9}\left(2 \Pi_{I 0}^{\prime}+\Pi_{I I 0}^{\prime}\right)\right] \\
& +\frac{(1+2 \varphi)}{r \sqrt{2}}\left(3 b-a+2 a \varphi-3 b \varphi-\frac{3 l}{r}\right) \\
& +\frac{l}{2 r} X_{10 p N}+\frac{b(1-\varphi)}{2} X_{30 p N}+\Upsilon_{p N}+\Phi_{p N}+\zeta_{p N}, \\
\zeta_{2}= & -\frac{r^{3}(1-\varphi)}{2 \sqrt{2}} \frac{\Pi_{K N 0} \chi_{p N}}{\mu_{0}+\Pi_{K N 0}} \\
& \times\left[6(1+\varphi)_{\theta}(1-\varphi)+(1+\varphi)_{\theta}(1+\varphi)+2(1+\varphi)\right. \\
& \left.\times\left\{(1-\varphi)_{\theta}(1+\varphi)+(1+\varphi)_{\theta}(1-\varphi)\right\}\right] \\
& +\frac{(1+2 \varphi)}{2 \sqrt{2}}\left(\frac{\Pi_{K N 0} \chi_{p N}}{\mu_{0}+\Pi_{K N 0}}\right)_{\theta},
\end{aligned}
$$

and the subscript $\mathrm{pN}$ represents the effects of the quantities at the $\mathrm{pN}$ era. The quantity $\Upsilon_{p N}$ describes the reflection effects of the non-static axial celestial body about its symmetry axis at $\mathrm{pN}$ approximations. It is worth mentioning here that these constraints coincide with those of [39] in the limit $L \rightarrow 0$ for $n=2$.

\section{Instability of realistic star object}

Perturbations of stars and black holes have been among the main topics of relativistic astrophysics for the last few decades. The description of such stellar objects has recently attracted various researchers [55-60]. The stability analysis of the general relativistic star process is an important but challenging endeavor. In such a study, the spherical symmetric matter configuration is an exemplary one. Numerous realistic objects like globular clusters, galactic bulges, and dark matter halos can be considered to be of a roughly spherical geometry. For better understanding of the cosmic censorship hypothesis and the hoop conjecture, it is necessary to shed light on the non-spherical collapse. The physical interest in studying non-spherical symmetries is associated with the fact that post-shocked clouds are left at the verge of gravitational collapse forming cylinders or plates at scales of galaxy formation and at scales of stellar formation in a galaxy. For 
instance, cylindrical distributions are closely related with the problem of fragmentation of prestellar clouds [61].

We take into account a specific configuration of a nonstatic axial spacetime. The main purpose is to study instability regimes of axially symmetric realistic objects that are involved in the emission of gravitational radiation due to meridional degrees of freedom. For this purpose, we assume coupling of the system with an anisotropic fluid distribution whose energy-momentum tensor is mentioned in Eq. (5). Having arrived at this point, the relevant question is: does an ideal (or non-anisotropic) matter configuration produce gravitational radiation?

To answer such a burning issue, let us recall that in the seminal paper of Bondi about the emission of gravitational radiation (Section 6 of [62]), it is mentioned that for a relativistic dust cloud as well as for the dissipation-less case of an ideal matter distribution, the relativistic system cannot be anticipated to radiate (gravitationally). This is due to the reversible feature of the equation of state as emission of radiation is an irreversible phenomenon. This happens once absorption is considered (and/or taking Sommerfeld type constraints), which prevents the inflow of waves. This implies that an entropy generator parameter/factor must be present in the discussion of a relativistic source. However, such a type of factor is not present in an ideal fluid and in a collisionless dust cloud. In particular, the irreversibility of gravitational wave emissions must be taken in the equation of state with the help of an entropy increasing (dissipative) parameter. In this scenario, Herrera et al. [63] described a close relationship between vorticity and gravitational radiation.

We consider the evolution of a non-static axisymmetric self-gravitating system in $f(R)$ gravity and assume that it is in hydrostatic equilibrium at large past time. Now we want to analyze the phase of equilibrium being disturbed. Will this perturbation be relaxed (stable state) or will it grow (unstable state)? In this respect, one needs to take into account the following instabilities:

1. Dynamical stability: what happens, if the stellar hydrostatic phase is perturbed?

2. Secular (thermal) stability: what happens, when the state of thermal equilibrium is perturbed?

Since our system is coupled with anisotropic matter configurations without heat flux, we shall not discuss the second case and confine ourselves to the dynamical instability of relativistic origin. It is seen that for a hydrostatic phase, the stability criterion is achieved by making a linearized field as well as conservation equations against the radial perturbation (14)-(17). It is noted that, during evolution, the realistic object moves via several evolutionary patterns determined by instability/stability degrees of freedom. This suggests that the relativistic systems can be stable at one instance and not at the other. Thus one needs to cope with the dynamical evolution of self-gravitating systems by calculating the instability regions at the $\mathrm{N}$ as well as the $\mathrm{pN}$ regimes. Such epochs have a vital role to play in the discussion of gravitational collapse of compact objects.

The phenomenon of celestial collapse occurs when the state of hydrostatic equilibrium of a stellar object is disturbed. In celestial body, nuclear fission reactions occur that start from hydrogen atoms and produce further complex elements until a nuclear reactions chain stops with iron. These reactions increase the pressure exerted by gas particles which counterbalances the gravitational attraction and prevents the star from collapsing. However, with the passage of time, nuclear reactions decrease as the fuel burns out. Consequently, the necessary pressure becomes insufficient for a collapsing body to be stable. At this point, the gravitational force begins to pull matter toward the center of a body and thus collapse initiates. A celestial body that has exhausted all its nuclear fuel can give birth to three possible compact objects (white dwarfs, neutron stars, and black holes) on the basis of the initial mass of the collapsing body.

It is well known that, in the scenario of the Newtonian regime, the instability of spherical self-gravitating systems depends purely on the mean value of the stiffness parameter, $\Gamma_{1}$ [64], which is the ratio of the fractional Lagrangian variations between pressure and energy density experienced by matter configurations following the motion. However, in GR, the stability relies not only on the average value of $\Gamma_{1}$ but also on the radius of the star. However, in the study of a non-static axial reflection system in modified gravity, the situation is quite different. (It is worthy to stress that we have assumed $\Gamma_{1}$ to be a constant entity throughout the analysis.) The most important consequence of our study is that, apart from affirming the GR results, $\Gamma_{1}$ controls the emission of gravitational radiation along with the $f(R)$ extra degrees of freedom. The emission of gravitational radiation causes the loss of both energy and angular momentum, which increases the instability of the meridional axisymmetric object.

More specifically, following the results of Chandrasekhar [25], we deduce that if the anisotropic matter distribution attains a stiffness equal to the right hand side of Eqs. (34) and (35), the system enters into the window of the hydrostatic equilibrium at the $\mathrm{N}$ and $\mathrm{pN}$ regimes. Further, if the stiffness of the fluid increases in such a way that the fractional value given at the right hand side of (34) and (35) becomes a smaller one, then the system enters into the stable configurations at both the $\mathrm{N}$ and the $\mathrm{pN}$ approximations, respectively. Dosopoulou et al. [65] explored the contribution of magnetic fields in the emergence and existence of vorticity. This strongly suggests that invoking magnetic fields in 
the study of the stability of gravitationally radiating sources deserves attention for future work.

\section{Conclusions}

It is well known that the most general non-static axial geometry incorporates reflection (meridional) and rotation effects coming from the non-diagonal $\mathrm{d} t \mathrm{~d} \theta$ and $\mathrm{d} t \mathrm{~d} \phi$ metric coefficients. In order to deal analytically with the instability constraints of axially symmetric spacetime, several attempts have been made by taking a restricted class of axial geometry. In this paper, we have made a stability analysis of the meridional axial stellar structure with a $f(R)$ background. We perform an investigation in a metric $f(R)$ gravity which give rise to non-linear fourth order field equations. We have formulated the collapse equation by using a perturbation scheme in the generalized Euler equation. We assume a complete hydrostatic equilibrium of the axial stellar structure at large past time, i.e., $T(-\infty)=0$.

We have developed instability constraints at the $\mathrm{N}$ and the $\mathrm{pN}$ epochs through the stiffness parameter, $\Gamma_{1}$, using the collapse equation. It is found that the axial stellar structure would be unstable until it obeys Eq. (34) at the $\mathrm{N}$ regime, while we have Eq. (35) at the $\mathrm{pN}$ era. Breaching of these inequalities will eventually move the system toward a stable window. These constraints depend upon the adiabatic index, and static combinations of anisotropy, energy density, and dark source corrections due to the $R+\epsilon R^{n}$ model. It is seen that dark source corrections tend to stabilize the structure formation phenomenon due to its non-attractive behavior, while the presence of non-diagonal terms in the instability ranges indicate the occurrence of gravitational radiation, which corresponds to the flow of super-energy [24].

We have found the non-vanishing component of vorticity tensor which corresponds to a non-static meridional axial structure coefficient. The inclusion of a non-diagonal scale factor in the stability analysis leads to the interesting phenomenon of gravitational radiation for $\epsilon R^{n}$ corrections. These extra-order $f(R)$ corrections affect the passive gravitational mass, which in turn affects the rate of stellar collapse. We have developed the instability constraints (34) and (35) with the weak field and $\mathrm{pN}$ approximations, respectively. These constraints can be applied to an axisymmetric selfgravitating system with reflection degrees around the symmetry axis at some particular cosmic epochs, depending upon the chosen values of $n$. We can categorize different eras of the cosmic dynamics associated with $\epsilon R^{n}$ as follows:

- For $n=2$, the instability constraints for the specific model of the type $R+\epsilon R^{2}$ can be obtained. The existence of the $R^{2}$ correction in the field equations can be helpful to explain the inflationary mechanism of the cos- mos. The term $\alpha R^{2}$ represents an accelerated expansion of the universe. This model is compatible with temperature anisotropies observed in cosmic microwave background radiation [43-46] and hence is viable for inflationary scalar field models.

- The choice $n=3$ favors significantly massive compact objects coming from cubic $f(R)$ higher curvature terms [36]. This provides a realistic signature of the presence of more massive and huge self-gravitating stellar systems, which have a direct correspondence with the observational cosmology.

- This gravitational dynamics at a late-time universe era can be obtained by substituting $n=-1$ in the instability constraints at both the $\mathrm{N}$ and the $\mathrm{pN}$ regimes. It is noticed that the gravitational contribution due to a negative curvature power serves as dark energy, thereby supporting the current accelerating cosmic epochs [42].

- For $\epsilon=0$, instability constraints for Einstein gravity can be obtained at both the $\mathrm{N}$ and the $\mathrm{pN}$ eras, which describe a relatively less stable axial stellar structure.

Finally, we remark that supermassive stellar systems survive more abundantly in extended gravity than in GR, as such theories (for instance $f(R)$ gravity) are more likely to host huge stars with smaller radii. This leads to the existence of more dense relativistic systems, which have direct relevance for observational gravitational physics. It is interesting to mention here that all our results reduce to a restricted class of instability analysis [39] by neglecting non-diagonal terms and assuming $n=2$ in the $f(R)$ model.

Acknowledgments We would like to thank the Higher Education Commission, Islamabad, Pakistan, for its financial support through the Indigenous Ph.D. Fellowship for 5 K Scholars, Phase-II, Batch-I.

Open Access This article is distributed under the terms of the Creative Commons Attribution 4.0 International License (http://creativecomm ons.org/licenses/by/4.0/), which permits unrestricted use, distribution, and reproduction in any medium, provided you give appropriate credit to the original author(s) and the source, provide a link to the Creative Commons license, and indicate if changes were made.

Funded by $\mathrm{SCOAP}^{3}$.

\section{Appendix A}

The extra $f(R)$ curvature terms for Eqs. (10)-(12) are

$$
\begin{aligned}
D_{0} & =\dot{V}_{1}+X_{1}^{\prime}+X_{3}^{\prime} \\
& +\left(\frac{3 B^{2} r^{2} A A^{\prime}}{\Delta}+\frac{2 A^{2} B^{2} r}{\Delta}+\frac{2 r^{2} A^{2} B B^{\prime}}{\Delta}+\frac{C^{\prime}}{C}\right. \\
& \left.+\frac{B^{\prime}}{B}\right) X_{1}+\left(\frac{3 r^{2} B^{2} A A_{\theta}}{\Delta}+\frac{B_{\theta}}{B}+\frac{C_{\theta}}{C}\right. \\
& \left.+\frac{A^{2} r^{2} B B_{\theta}}{\Delta}\right) X_{3}+\left(\frac{r^{2} B^{3} \dot{B}}{\Delta}+\frac{B^{\prime}}{B}\right) V_{2}
\end{aligned}
$$




$$
\begin{aligned}
& +\frac{r^{2} B^{2}}{\Delta}\left(r^{2} B \dot{B} V_{3}+C \dot{C} V_{4}\right)+\dot{W}_{1}+\left(Y_{1}+Y_{3}\right)^{\prime} \\
& +\frac{L W_{1}}{\Delta}\left(A A_{\theta}+L \dot{L}\right)+\frac{L \dot{L} V_{1}}{\Delta} \\
& +\left(\frac{3 r^{2} B^{2} A A^{\prime}}{\Delta}+\frac{B^{\prime}}{B}+\frac{2 r A^{2} B^{2}}{\Delta}+\frac{2 r^{2} A^{2} B B^{\prime}}{\Delta}\right. \\
& \left.+\frac{C^{\prime}}{C}+\frac{4 L L^{\prime}}{\Delta}\right) Y_{1}\left(\frac{B_{\theta}}{B} \times \frac{3 r^{2} B^{2} A A_{\theta}}{\Delta}+\frac{r^{2} A^{2} B B_{\theta}}{\Delta}\right. \\
& +\frac{C_{\theta}}{C}+\frac{3 L r^{2} B \dot{B}}{\Delta}-\frac{r^{2} B^{2} L^{\prime}}{2 \Delta}+\frac{r L B^{2}}{\Delta}+\frac{r^{2} L B B^{\prime}}{\Delta} \\
& \left.-\frac{r^{2} L B \dot{B}}{\Delta}+\frac{L L_{\theta}}{\Delta}\right) Y_{3}+\left(\frac{3 r^{2} L B \dot{B}}{\Delta}-\frac{r^{2} B^{2} L^{\prime}}{2 \Delta}\right. \\
& \left.+\frac{r^{2} L B B^{\prime}}{\Delta}+\frac{r B^{2} L}{\Delta}+\frac{L L_{\theta}}{\Delta}-\frac{r^{2} L B \dot{B}}{\Delta}\right) X_{3} \\
& +\left(\frac{r^{2} B^{3} \dot{B}}{\Delta}+\frac{B^{\prime}}{B}-\frac{L B B_{\theta}}{\Delta}\right) W_{2} \\
& -\frac{L B B_{\theta}}{\Delta} V_{2}+\left(\frac{r L B^{2}}{\Delta}+\frac{r^{2} B L B^{\prime}}{\Delta}-\frac{r^{2} B^{2} L^{\prime}}{2 \Delta}\right)\left(X_{2}+Y_{2}\right) \\
& +\left(\frac{r B L B_{\theta}}{\Delta}-\frac{r^{2} B^{2} L_{\theta}}{\Delta}+\frac{r^{4} B^{3} \dot{B}}{\Delta}\right) W_{3} \\
& +\left(\frac{r B L B_{\theta}}{\Delta}-\frac{r^{2} B^{2} L_{\theta}}{\Delta}\right) V_{3}+\left(\frac{r^{2}}{\Delta} B^{2} C \dot{C}-\frac{L C C_{\theta}}{\Delta}\right) \\
& \times W_{4}-\frac{L C C_{\theta}}{\Delta} V_{4}, \\
& D_{1}=\dot{X}_{1}-\left(r+\frac{r^{2} B^{\prime}}{B}\right) W_{3}-\frac{C C^{\prime}}{B^{2}} W_{4}+X_{2 \theta}+V_{2}^{\prime} \\
& \times \frac{A A^{\prime}}{B^{2}} V_{1}+\left(\frac{2 B^{\prime}}{B}+\frac{r^{2} B^{2} A A^{\prime}}{\Delta}+\frac{2 r A^{2} B^{2}}{\Delta}\right. \\
& \left.+\frac{2 r^{2} A^{2} B B^{\prime}}{\Delta}+\frac{C^{\prime}}{C}\right) V_{2}+\left(\frac{3 B_{\theta}}{B}+\frac{r^{2} B^{2} A A_{\theta}}{\Delta}\right. \\
& \left.+\frac{r^{2} A^{2} B B_{\theta}}{\Delta}+\frac{C_{\theta}}{C}\right) X_{2}+\frac{A^{2} r^{2} B \dot{B}}{\Delta} X_{3} \\
& D_{2}=\dot{X}_{3}+X_{2}^{\prime}+V_{3 \theta}+\frac{A^{3} A_{\theta}}{\Delta} V_{1}-\frac{A^{2} B B_{\theta}}{\Delta} V_{2} \\
& -\frac{A^{2} C C_{\theta}}{\Delta} V_{4}+\left(\frac{r^{2} B^{2} A A_{\theta}}{\Delta}+\frac{2 r^{2} A^{2} B B_{\theta}}{\Delta}+\frac{B_{\theta}}{B}\right. \\
& \left.+\frac{C_{\theta}}{C}\right) V_{3}+\left(\frac{3 A^{2} r^{2} B \dot{B}}{\Delta}-\frac{2 A L A_{\theta}}{\Delta}+\frac{r^{2} B^{2} A \dot{A}}{\Delta}\right. \\
& \left.+\frac{\dot{B}}{B}+\frac{\dot{C}}{C}\right) X_{3}+Y_{2}^{\prime}+\dot{Y}_{3} \\
& +\left(\frac{6 r A^{2} B^{2}}{\Delta}+\frac{6 r^{2} A^{2} B B^{\prime}}{\Delta}+\frac{2 r^{2} B^{2} A A^{\prime}}{\Delta}+\frac{B^{\prime}}{B}\right. \\
& \left.+\frac{C^{\prime}}{C}\right) X_{2}+W_{3 \theta}+\left(\frac{A^{3} A_{\theta}}{\Delta}+\frac{A^{2} \dot{L}}{\Delta}-\frac{A L \dot{A}}{\Delta}\right) W_{1} \\
& +\left(\frac{A^{2} \dot{L}}{\Delta}-\frac{A L \dot{A}}{\Delta}\right) V_{1}-\left(\frac{A^{2} B B_{\theta}}{\Delta}-\frac{B L \dot{B}}{\Delta}\right) W_{2} \\
& -\frac{B L \dot{B}}{\Delta} V_{2}-\left(\frac{A^{2}}{\Delta} C C_{\theta}+\frac{C L \dot{C}}{\Delta}\right) W_{4} \\
& -\frac{C L \dot{C}}{\Delta} V_{4}+\left(\frac{r^{2} B^{2} A A_{\theta}}{\Delta}+\frac{2 r^{2} A^{2} B B_{\theta}}{\Delta}+\frac{r^{2} B L \dot{B}}{\Delta}\right. \\
& \left.+\frac{2 L L_{\theta}}{\Delta}+\frac{B_{\theta}}{B}+\frac{C_{\theta}}{C}-\frac{2 r^{2} L B \dot{B}}{\Delta}\right) W_{3} \\
& +\frac{L V_{3}}{\Delta}\left(r^{2} B L \dot{B}+2 L_{\theta}-2 r^{2} B \dot{B}\right) \\
& +\left(\frac{A^{2} L^{\prime}}{\Delta}-\frac{2 A L A^{\prime}}{\Delta}\right) \\
& \times\left(X_{1}+Y_{1}\right)+\left(\frac{3 A^{2} r^{2} B \dot{B}}{\Delta}-\frac{2 A L A_{\theta}}{\Delta}+\frac{r^{2} B^{2} A \dot{A}}{\Delta}\right. \\
& \left.+\frac{\dot{B}}{B}+\frac{\dot{C}}{C}+\frac{L \dot{L}}{\Delta}\right) Y_{3}+\left(\frac{L \dot{L}}{\Delta}-\frac{2 L A A_{\theta}}{\Delta}\right) X_{3} \\
& +\left(\frac{6 r A^{2} B^{2}}{\Delta}+\frac{6 r^{2} A^{2} B B^{\prime}}{\Delta}+\frac{2 r^{2} B^{2} A A^{\prime}}{\Delta}+\frac{B^{\prime}}{B}\right. \\
& \left.+\frac{C^{\prime}}{C}+\frac{7 L L^{\prime}}{2 \Delta}\right) Y_{2}+\frac{7 L L^{\prime}}{2 \Delta} X_{2} \text {. }
\end{aligned}
$$$$
-\left(r+\frac{r^{2} B^{\prime}}{B}\right) V_{3}-\frac{C C^{\prime} V_{4}}{B^{2}}+\dot{Y}_{1}+W_{2}^{\prime}+Y_{2 \theta}
$$$$
+\left(\frac{3 \dot{B}}{B}+\frac{r^{2} B^{2} A \dot{A}}{\Delta}+\frac{\dot{C}}{C}+\frac{L A A_{\theta}}{\Delta}+\frac{L \dot{L}}{\Delta}\right) Y_{1}
$$$$
+\left(\frac{L A A_{\theta}}{\Delta}+\frac{L \dot{L}}{\Delta}\right) X_{1}+\left(\frac{2 B^{\prime}}{B}+\frac{r^{2} B^{2} A A^{\prime}}{\Delta}\right.
$$$$
\left.+\frac{2 r A^{2} B^{2}}{\Delta}+\frac{2 r^{2} A^{2} B B^{\prime}}{\Delta}+\frac{C^{\prime}}{C}+\frac{3 L L^{\prime}}{\Delta}\right) W_{2}
$$$$
+\frac{3 L L^{\prime}}{\Delta} V_{2}+\left(\frac{3 B_{\theta}}{B}+\frac{r^{2} B^{2} A A_{\theta}}{\Delta}+\frac{r^{2} B^{2} B B_{\theta}}{\Delta}+\frac{C_{\theta}}{C}\right.
$$$$
\left.+\frac{L L_{\theta}}{\Delta}\right) Y_{2}+\frac{L L_{\theta}}{\Delta} X_{2}+\left(\frac{r^{2} A^{2} B \dot{B}}{\Delta}-\frac{L^{\prime}}{B}\right.
$$$$
\left.-\frac{L A A_{\theta}}{\Delta}\right) Y_{3}-\left(\frac{L^{\prime}}{B}+\frac{L A A_{\theta}}{\Delta}\right) X_{3},
$$

The perturbed parts of Eq. (10) are

$$
\begin{aligned}
g= & {\left[x_{1}^{\prime}+x_{1}\left(\frac{3 B_{0}^{2} r^{2} A_{0} A_{0}^{\prime}}{\Delta_{0}}+\frac{B_{0}^{\prime}}{B_{0}}+\frac{2 A_{0}^{2} B_{0}^{2} r}{\Delta_{0}}\right.\right.} \\
& \left.+\frac{2 A_{0}^{2} r^{2} B_{0} B_{0}^{\prime}}{\Delta_{0}}+\frac{C_{0}^{\prime}}{C_{0}}\right)+X_{10}\left\{\frac { 3 B _ { 0 } ^ { 2 } r ^ { 2 } A _ { 0 } A _ { 0 } ^ { \prime } } { \Delta _ { 0 } } \left(\frac{2 b}{B_{0}}\right.\right. \\
& \left.+\frac{a}{A_{0}}+\frac{a^{\prime}}{A_{0}^{\prime}}-\frac{d}{\Delta_{0}}\right)+\left(\frac{b}{B_{0}}\right)^{\prime}+\frac{2 A_{0}^{2} B_{0}^{2} r}{\Delta_{0}}\left(\frac{2 a}{A_{0}}\right. \\
& \left.+\frac{2 b}{B_{0}}-\frac{d}{\Delta_{0}}\right)+\frac{2 A_{0}^{2} r^{2} B_{0} B_{0}^{\prime}}{\Delta_{0}}\left(\frac{2 a}{A_{0}}+\frac{b}{B_{0}}+\frac{b^{\prime}}{B_{0}^{\prime}}\right) \\
& \left.+\left(\frac{c}{C_{0}}\right)^{\prime}\right\}+x_{3}\left(\frac{3 r^{2} B_{0}^{2} A_{0} A_{0 \theta}}{\Delta_{0}}\right.
\end{aligned}
$$




$$
\begin{aligned}
& \left.+\frac{C_{0 \theta}}{C_{0}}+\frac{B_{0 \theta}}{B_{0}}+\frac{A_{0}^{2} r^{2} B_{0}^{2} B_{0 \theta}}{\Delta_{0}}\right) \\
& +X_{30}\left\{\frac{r B_{0}^{2} L_{0}}{\Delta_{0}}\left(\frac{2 b}{B_{0}}-\frac{d}{\Delta_{0}}\right)\right. \\
& +X_{30}\left\{\frac{3 r^{2} B_{0}^{2} A_{0} A_{0 \theta}}{\Delta_{0}}\left(\frac{2 b}{B_{0}}+\frac{a}{A_{0}}+\frac{a_{\theta}}{A_{0 \theta}}-\frac{d}{\Delta_{0}}\right)\right. \\
& -\frac{r^{2} B_{0}^{2} L_{0}^{\prime}}{2 \Delta_{0}}\left(\frac{2 b}{B_{0}}+\frac{l^{\prime}}{L_{0}^{\prime}}-\frac{d}{\Delta_{0}}\right)+\frac{r^{2} B_{0} B_{0}^{\prime} L_{0}}{\Delta_{0}} \\
& +\left(\frac{c}{C_{0}}\right)_{\theta}+\left(\frac{c}{C_{0}}\right)_{\theta}+\frac{A_{0}^{2} r^{2} B_{0}^{2} B_{0 \theta}}{\Delta_{0}} \\
& \times\left(\frac{b}{B_{0}}+\frac{l}{L_{0}}+\frac{b^{\prime}}{B_{0}^{\prime}}-\frac{d}{\Delta_{0}}\right) \\
& \left.\times\left(\frac{2 a}{A_{0}}+\frac{2 b}{B_{0}}+\frac{b_{\theta}}{B_{0 \theta}}\right)\right\} \\
& \left.+\frac{L_{0} L_{0 \theta}}{\Delta_{0}}\left(\frac{l}{L_{0}}+\frac{l_{\theta}}{L_{0 \theta}}-\frac{d}{\Delta_{0}}\right)\right\} \\
& +W_{10} \frac{L_{0} A_{0} A_{0 \theta}}{\Delta_{0}}\left(\frac{l}{L_{0}}+\frac{a}{A_{0}}+\frac{a_{\theta}}{A_{0 \theta}}-\frac{d}{\Delta_{0}}\right) \\
& +w_{2}\left(\frac{B_{0}^{\prime}}{B_{0}}-\frac{L_{0} B_{0} B_{0 \theta}}{\Delta_{0}}\right)+\left\{\left(\frac{b}{B_{0}}\right)^{\prime}-\frac{L_{0}}{\Delta_{0}}\right. \\
& +y_{1}\left(\frac{3 r^{2} B_{0}^{2} A_{0}^{2} B_{0 \theta}}{\Delta_{0}}+\frac{C_{0}^{\prime}}{C_{0}}+\frac{B_{0}^{\prime}}{B_{0}}\right. \\
& \left.+\frac{2 A_{0}^{2} B_{0}^{2} r}{\Delta_{0}}+\frac{2 A_{0}^{2} B_{0} r^{2} B_{0}^{\prime}}{\Delta_{0}}+\frac{4 L_{0} L_{0}^{\prime}}{\Delta_{0}}\right) \\
& +Y_{10}\left\{\frac { 3 r ^ { 2 } B _ { 0 } ^ { 2 } A _ { 0 } ^ { 2 } B _ { 0 \theta } } { \Delta _ { 0 } } \left(\frac{2 b}{B_{0}}+\frac{a}{A_{0}}+\frac{a^{\prime}}{A_{0}^{\prime}}\right.\right. \\
& \left.-\frac{d}{\Delta_{0}}\right)+\left(\frac{b}{B_{0}}\right)^{\prime}+\frac{2 A_{0}^{2} B_{0}^{2} r}{\Delta_{0}} \\
& \times\left(\frac{2 a}{A_{0}}+\frac{2 b}{B_{0}}-\frac{d}{\Delta_{0}}\right)+\frac{2 A_{0}^{2} B_{0} r^{2} B_{0}^{\prime}}{\Delta_{0}}\left(\frac{2 a}{A_{0}}+\frac{b}{B_{0}}\right. \\
& \left.+\frac{b^{\prime}}{B_{0}^{\prime}}-\frac{d}{\Delta_{0}}\right)+\left(\frac{c}{C_{0}}\right)^{\prime}+\frac{4 L_{0} L_{0}^{\prime}}{\Delta_{0}}\left(\frac{l}{L_{0}}+\frac{l^{\prime}}{L_{0}^{\prime}}\right. \\
& \left.\left.-\frac{d}{\Delta_{0}}\right)\right\}+\frac{4 x_{1} L_{0} L_{0}^{\prime}}{\Delta_{0}}+X_{10} \times \frac{4 L_{0} L_{0}^{\prime}}{\Delta_{0}}\left(\frac{l}{L_{0}}+\frac{l^{\prime}}{L_{0}}\right. \\
& \left.-\frac{d}{\Delta_{0}}\right)+y_{3}\left(\frac{3 r^{2} B_{0}^{2} A_{0} A_{0 \theta}}{\Delta_{0}}+\frac{C_{0 \theta}}{C_{0}}+\frac{A_{0}^{2} r^{2} B_{0} B_{0 \theta}}{\Delta_{0}}\right. \\
& \left.+\frac{B_{0 \theta}}{B_{0}}-\frac{r^{2} L_{0}^{\prime} B_{0}^{2}}{2 \Delta_{0}}+\frac{r L_{0} B_{0}^{2}}{\Delta_{0}}+\frac{r^{2} L_{0} B_{0} B_{0}^{\prime}}{\Delta_{0}}+\frac{L_{0} L_{0 \theta}}{\Delta_{0}}\right) \\
& +Y_{30}\left\{\frac{3 r^{2} B_{0}^{2} A_{0} A_{0 \theta}}{\Delta_{0}}\left(\frac{2 b}{B_{0}}+\frac{a}{A_{0}}+\frac{a_{\theta}}{A_{0 \theta}}-\frac{d}{\Delta_{0}}\right)\right. \\
& +\left(\frac{b}{B_{0}}\right)_{\theta}+\frac{A_{0}^{2} r^{2} B_{0} B_{0 \theta}}{\Delta_{0}}\left(\frac{b}{B_{0}}+\frac{2 a}{A_{0}}+\frac{b_{\theta}}{B_{0 \theta}}\right. \\
& \left.-\frac{d}{\Delta_{0}}\right)+\left(\frac{c}{C_{0}}\right)_{\theta}-\frac{r^{2} L_{0}^{\prime} B_{0}^{2}}{2 \Delta_{0}}\left(\frac{l^{\prime}}{L_{0}^{\prime}}+\frac{2 b}{B_{0}}-\frac{d}{\Delta_{0}}\right) \\
& +\frac{r L_{0} B_{0}^{2}}{\Delta_{0}}\left(\frac{l}{L_{0}}+\frac{2 b}{B_{0}}-\frac{d}{\Delta_{0}}\right) \\
& +\frac{r^{2} L_{0} B_{0} B_{0}^{\prime}}{\Delta_{0}}\left(\frac{l}{L_{0}}+\frac{b}{B_{0}}+\frac{b^{\prime}}{B_{0}^{\prime}}-\frac{d}{\Delta_{0}}\right) \\
& \left.+\frac{L_{0} L_{0 \theta}}{\Delta_{0}}\left(\frac{l}{L_{0}}+\frac{l_{\theta}}{L_{0 \theta}}-\frac{d}{\Delta_{0}}\right)\right\}+x_{3} \\
& \times\left(\frac{r B_{0}^{2} L_{0}}{\Delta_{0}}-\frac{r^{2} B_{0}^{2} L_{0}^{\prime}}{2 \Delta_{0}}+\frac{r^{2} B_{0} B_{0}^{\prime} L_{0}}{\Delta_{0}}+\frac{L_{0} L_{0 \theta}}{\Delta_{0}}\right) \\
& \left.\times B_{0} B_{0 \theta}\left(\frac{l}{L_{0}}+\frac{b}{B_{0}}+\frac{b_{\theta}}{B_{0 \theta}}-\frac{d}{\Delta_{0}}\right)\right\} \\
& \times W_{20}-V_{20} \frac{L_{0} B_{0} B_{0 \theta}}{\Delta_{0}}\left(\frac{l}{L_{0}}+\frac{b}{B_{0}}+\frac{b_{\theta}}{B_{0 \theta}}-\frac{d}{\Delta_{0}}\right) \\
& -v_{2} \frac{L_{0} B_{0} B_{0 \theta}}{\Delta_{0}}+\left(x_{2}+y_{2}\right) \\
& \times\left(\frac{r}{\Delta_{0}} L_{0} B_{0}^{2}+\frac{r^{2} B_{0} B_{0}^{\prime}}{\Delta_{0}}-\frac{r^{2} B_{0}^{2} L_{0}^{\prime}}{2 A_{0}}\right) \\
& +\left(x_{2}+y_{2}\right)\left\{\frac{r L_{0} B_{0}^{2}}{\Delta_{0}}\left(\frac{l}{L_{0}}+\frac{2 b}{B_{0}}-\frac{d}{\Delta_{0}}\right)\right. \\
& +\frac{r^{2} B_{0} B_{0}^{\prime}}{\Delta_{0}}\left(\frac{l}{L_{0}}+\frac{b}{B_{0}}+\frac{b^{\prime}}{B_{0}^{\prime}}\right. \\
& \left.\left.-\frac{d}{\Delta_{0}}\right)-\frac{r^{2} B_{0}^{2} L_{0}^{\prime}}{2 A_{0}}\left(\frac{l^{\prime}}{L_{0}^{\prime}}+\frac{2 b}{B_{0}}-\frac{d}{\Delta_{0}}\right)\right\} \\
& +\left(w_{3}+v_{3}\right)\left(\frac{r}{\Delta_{0}} B_{0} L_{0} B_{0 \theta}\right. \\
& \left.-\frac{r^{2} B_{0}^{2} L_{0 \theta}}{\Delta_{0}}\right)+\left(W_{30}+V_{30}\right) \\
& \times\left\{\frac{r B_{0} L_{0} B_{0 \theta}}{\Delta_{0}}\left(\frac{l}{L_{0}}+\frac{b}{B_{0}}+\frac{b_{\theta}}{B_{0 \theta}}-\frac{d}{\Delta_{0}}\right)-\left(\frac{2 b}{B_{0}}\right.\right. \\
& \left.\left.+\frac{a_{\theta}}{A_{0 \theta}}-\frac{d}{\Delta_{0}}\right) \frac{r^{2} B_{0}^{2} L_{0 \theta}}{\Delta_{0}}\right\} \\
& -\left(v_{4}+w_{4}\right) \frac{L_{0} C_{0} C_{0 \theta}}{\Delta_{0}}-\left(V_{40}+W_{40}\right) \frac{L_{0} C_{0} C_{0 \theta}}{\Delta_{0}} \\
& \times\left(\frac{l}{L_{0}}+\frac{c}{C_{0}}+\frac{c_{\theta}}{C_{0 \theta}}-\frac{d}{\Delta_{0}}\right)+V_{20}\left(\frac{b}{B_{0}}\right)^{\prime} \\
& \left.+v_{2} \frac{B_{0}^{\prime}}{B_{0}}+\frac{w_{1} L_{0} A_{0} A_{0 \theta}}{\Delta_{0}}\right] T \text {, } \\
& h=v_{1}+V_{10}\left(\frac{B_{0}^{2} r^{2} A_{0} a}{\Delta_{0}}+\frac{c}{C_{0}}\right)+\frac{l L_{0}}{\Delta_{0}}\left(V_{10}+W_{10}\right) \\
& +\frac{b B_{0}^{3} r^{2}}{\Delta_{0}}\left(V_{20}+W_{20}\right)+\frac{b B_{0}^{3} r^{4}}{\Delta_{0}}\left(V_{30}+W_{30}\right)
\end{aligned}
$$


$+\frac{c C_{0} B_{0}^{2} r^{2}}{\Delta_{0}}\left(V_{40}+W_{40}\right)+w_{1}+\frac{L_{0}^{2} l W_{10}}{\Delta_{0}}$.

The perturbed parts of Eq. (23) are

$$
\begin{aligned}
& \zeta=\frac{r^{3} A_{0} B_{0}^{3}}{\Delta_{0}^{\frac{3}{2}}}\left(\frac{a}{A_{0}}+\frac{3 b}{B_{0}}-\frac{3 d}{\Delta_{0}}\right) \\
& \times\left\{\frac{A_{0 \theta}}{A_{0}}+\frac{6 B_{0 \theta}}{B_{0}}+\frac{C_{0 \theta}}{C_{0}}+\frac{4 r^{2} A_{0}^{2} B_{0}^{2}}{\Delta_{0}}\left(\frac{A_{0 \theta}}{A_{0}}+\frac{B_{0 \theta}}{B_{0}}\right)\right\} . \\
& +\frac{r^{3} A_{0} B_{0}^{3}}{\Delta_{0}^{3 / 2}} \Pi_{K L 0}\left[\frac{6 B_{0 \theta}}{B_{0}}\left(\frac{b_{\theta}}{B_{0 \theta}}+\frac{b}{B_{0}}\right)+\left(\frac{a}{A_{0}}\right)_{\theta}\right. \\
& +\left(\frac{c}{C_{0}}\right)_{\theta}+\frac{4 r^{2} A_{0}^{2} B_{0}^{2}}{\Delta_{0}^{2}}\left(\frac{2 a}{A_{0}}+\frac{2 b}{B_{0}}-\frac{2 d}{\Delta_{0}}\right)\left(\frac{a}{A_{0}}\right. \\
& \left.\left.+\frac{b}{B_{0}}\right)_{\theta}\right]-\frac{\mu_{0} r^{4} A_{0}^{4}}{\Delta_{0}^{2}}\left(\frac{a}{A_{0}}\right)^{\prime}-\left\{P_{0}+\frac{2}{9}\left(2 \Pi_{I 0}\right.\right. \\
& \left.\left.+\Pi_{I I 0}\right)\right\} \frac{1}{B_{0}^{2}}\left[\left(\frac{c}{C_{0}}\right)^{\prime}+\frac{r^{2} A_{0}^{2} B_{0}^{2}}{Z_{0}^{2}}\left(\frac{2 a}{A_{0}}+\frac{2 b}{B_{0}}\right.\right. \\
& \left.\left.-\frac{2 d}{\Delta_{0}}\right)\left(\frac{A_{0}^{\prime}}{A_{0}}+\frac{1}{r}\right)+\frac{r^{2} A_{0}^{2} B_{0}^{2}}{\Delta_{0}}\left(\frac{a}{A_{0}}+\frac{b}{B_{0}}\right)^{\prime}\right] \\
& -\frac{2 b}{B_{0}^{2}}\left\{P_{0}+\frac{2}{9}\left(2 \Pi_{I 0}+\Pi_{I I 0}\right)\right\} \\
& \times\left[\frac{C_{0}^{\prime}}{C_{0}}+\frac{r^{2} A_{0}^{2} B_{0}^{2}}{\Delta_{0}}\left(\frac{A_{0}^{\prime}}{A_{0}}+\frac{1}{r}\right)\right],
\end{aligned}
$$$$
\Upsilon=-\left[\frac{4 r^{3} A_{0} L_{0} B_{0}^{3} L_{0 \theta}^{\prime}}{\Delta^{5 / 2}}\left(\frac{a}{A_{0}}+\frac{3 b}{B_{0}}-\frac{3 d}{\Delta_{0}}\right)\right.
$$$$
+\frac{4 L_{0} L_{0 \theta}}{\Delta}\left(\frac{l}{L_{0}}+\frac{l_{\theta}}{L_{0 \theta}}-\frac{d}{\Delta_{0}}\right)-\frac{A_{0 \theta} L_{0}}{r^{2} A_{0} B_{0}^{2}}
$$$$
\times\left(\frac{l}{L_{0}}+\frac{a_{\theta}}{A_{0 \theta}}-\frac{a}{A_{0}}-\frac{2 b}{B_{0}}\right)+\frac{\mu_{0} L_{0}^{2} A_{0}^{2} r^{2}}{\delta_{0}^{2}}
$$$$
\times\left(\frac{2 l}{L_{0}}+\frac{2 a}{A_{0}}-\frac{2 d}{\Delta_{0}}\right)+\frac{\mu_{0} L_{0}^{2} A_{0}^{2} r^{2}}{\Delta_{0}^{2}}\left\{\frac{L_{0}^{\prime}}{2 L_{0}}\right.
$$$$
\left.\times\left(\frac{l^{\prime}}{L_{0}^{\prime}}-\frac{l}{L_{0}}\right)+\left(\frac{b}{B_{0}}\right)^{\prime}\right\}-\frac{3 L_{0} L_{0}^{\prime}}{2 B_{0}^{2} \Delta_{0}}
$$$$
\times\left\{P_{0}+\frac{2}{9}\left(2 \Pi_{I 0}+\Pi_{I I 0}\right)\right\}\left(\frac{l}{L_{0}}+\frac{l^{\prime}}{L_{0}^{\prime}}-\frac{d}{\Delta_{0}}\right)
$$$$
\left.+Y_{10}\left(\frac{3 b}{B_{0}}+\frac{a r^{2} A_{0} B_{0}^{2}}{\Delta_{0}}+\frac{c}{C_{0}}\right)+y_{1}\right]
$$$$
+\left[\frac{r^{2} B_{0} L_{0} b}{\Delta_{0}}+\frac{L_{0} L_{0 \theta}}{\Delta_{0}}\left(\frac{l}{L_{0}}+\frac{l_{\theta}}{L_{0 \theta}}-\frac{d}{\Delta_{0}}\right)\right.
$$$$
\left.-\frac{r^{2} L_{0} b B_{0}}{\Delta_{0}}\right] \omega X_{20}-\frac{\omega r^{2} A_{0}^{2} b B_{0} Y_{30}}{\Delta_{0}}+\frac{A_{0} L_{0} A_{0 \theta}}{\Delta_{0}}
$$$$
\times\left(\frac{a_{\theta}}{A_{0 \theta}}+\frac{a}{A_{0}}+\frac{l}{L_{0}}-\frac{d}{\Delta_{0}}\right) Y_{10}+\frac{L_{0}}{\Delta_{0}}
$$

$\times A_{0} A_{0 \theta}\left(y_{1}+x_{1}\right)+X_{10} \frac{L_{0} A_{0} A_{0 \theta}}{\Delta_{0}}\left(\frac{a_{\theta}}{A_{0 \theta}}+\frac{a}{A_{0}}+\frac{l}{L_{0}}\right.$

$\left.-\frac{d}{\Delta_{0}}\right)+w_{2}\left(\frac{2 B_{0}^{\prime}}{B_{0}}+\frac{B_{0}^{2} r^{2} A_{0}}{\Delta_{0}}\right.$

$\left.\times A_{0}^{\prime}+\frac{2 r A_{0}^{2} B_{0}^{2}}{\Delta_{0}}+\frac{2 A_{0}^{2} r^{2} B_{0} B_{0}^{\prime}}{\Delta_{0}}+\frac{C_{0}^{\prime}}{C_{0}}+\frac{3 L_{0} L_{0}^{\prime}}{\Delta_{0}}\right)$

$+W_{20}\left\{2\left(\frac{b}{B_{0}}\right)^{\prime}+\frac{B_{0}^{2} r^{2} A_{0} A_{0}^{\prime}}{\Delta_{0}}\right.$

$\times\left(\frac{a}{A_{0}}+\frac{a^{\prime}}{A_{0}^{\prime}}+\frac{2 b}{B_{0}}-\frac{d}{\Delta_{0}}\right)+\frac{2 r A_{0}^{2} B_{0}^{2}}{\Delta_{0}}\left(\frac{2 b}{B_{0}}+\frac{2 a}{A_{0}}\right.$

$\left.-\frac{d}{\Delta_{0}}\right)+\frac{2 r^{2} A_{0}^{2} B_{0} B_{0}^{\prime}}{\Delta_{0}}\left(\frac{2 a}{A_{0}}+\frac{b^{\prime}}{B_{0}^{\prime}}+\frac{b}{B_{0}}-\frac{d}{\Delta_{0}}\right)$

$\left.+\left(\frac{c}{C_{0}}\right)^{\prime}+\frac{6 L_{0} L_{0}^{\prime}}{\Delta_{0}}\left(\frac{l}{L_{0}}-\frac{d^{\prime}}{\Delta_{0}}+\frac{l^{\prime}}{L_{0}^{\prime}}\right)\right\}$

$+\frac{3 w_{2} L_{0} L_{0}^{\prime}}{\Delta_{0}}+y_{2}$

$\times\left(\frac{3 B_{0 \theta}}{B_{0}}+\frac{C_{0 \theta}}{C_{0}}+\frac{r^{2} B_{0}^{2} A_{0} A_{0 \theta}}{\Delta_{0}}+\frac{r^{2} A_{0}^{2} B_{0} B_{0 \theta}}{\Delta_{0}}\right.$

$\left.+\frac{L_{0} L_{0 \theta}}{\Delta_{0}}\right)+y_{2 \theta}+Y_{20}\left\{3\left(\frac{b}{B_{0}}\right)_{\theta}+\frac{r^{2} B_{0}^{2} A_{0} A_{0 \theta}}{\Delta_{0}}\left(\frac{a}{A_{0}}\right.\right.$

$\left.+\frac{a_{\theta}}{A_{0 \theta}}+\frac{2 b}{B_{0}}-\frac{d}{\Delta_{0}}\right)+\frac{A_{0}^{2} r^{2} B_{0} B_{0 \theta}}{\Delta_{0}}\left(\frac{2 a}{A_{0}}+\frac{b}{B_{0}}+\frac{b_{\theta}}{B_{0 \theta}}\right.$

$\left.\left.-\frac{d}{\Delta_{0}}\right)+\left(\frac{c}{C_{0}}\right)_{\theta}+\frac{L_{0} L_{0 \theta}}{\Delta_{0}}\left(\frac{l}{L_{0}}+\frac{l_{\theta}}{L_{0 \theta}}-\frac{d}{\Delta_{0}}\right)\right\}$

$+x_{2} \frac{L_{0} L_{0 \theta}}{\Delta_{0}}-y_{3}\left(\frac{L_{0}^{\prime}}{B_{0}^{2}}+\frac{L_{0} A_{0} A_{0 \theta}}{\Delta_{0}}\right)$

$+w_{2}^{\prime}-Y_{30}\left\{\frac{L_{0}^{\prime}}{B_{0}^{2}}+\frac{L_{0} A_{0} A_{0 \theta}}{\Delta_{0}}\left(\frac{a}{A_{0}}+\frac{a_{\theta}}{A_{0 \theta}}\right.\right.$

$\left.\left.+\frac{l}{L_{0}}-\frac{d}{\Delta_{0}}\right)\right\}-r^{2} W_{30}\left(\frac{b}{B_{0}}\right)^{\prime}$

$-x_{3} \times\left(\frac{L_{0}^{\prime}}{B_{0}^{2}}+\frac{L_{0} A_{0} A_{0 \theta}}{\Delta_{0}}\right)-X_{30}$

$\times\left\{\frac{L_{0}^{\prime}}{B_{0}^{2}}\left(\frac{l^{\prime}}{L_{0}^{\prime}}-\frac{2 b}{B_{0}}\right)+\frac{L_{0} A_{0} A_{0 \theta}}{\Delta_{0}}\left(\frac{l}{L_{0}}+\frac{a}{A_{0}}\right.\right.$

$\left.\left.+\frac{a_{\theta}}{A_{0 \theta}}-\frac{d}{\Delta_{0}}\right)\right\}-w_{3}\left(r+\frac{r^{2} B_{0}^{\prime}}{B_{0}}\right)-w_{4} \frac{C_{0} C_{0}^{\prime}}{B_{0}^{2}}$

$+W_{40} \frac{C_{0} C_{0}^{\prime}}{B_{0}^{2}}\left(\frac{c}{C_{0}}+\frac{c^{\prime}}{C_{0}^{\prime}}-\frac{2 b}{B_{0}}\right)-\omega\left[2 y_{1}\right.$

$\left.+Y_{10}\left(\frac{3 b}{B_{0}}+\frac{a r^{2} A_{0} B_{0}^{2}}{\Delta_{0}}+\frac{c}{C_{0}}\right)\right]$, 


$$
\begin{aligned}
& \Phi=x_{2 \theta}+v_{2}^{\prime}+\frac{A_{0} A_{0}^{\prime}}{B_{0}}\left(\frac{a^{\prime}}{A_{0}^{\prime}}+\frac{a}{A_{0}}-\frac{2 b}{B_{0}}\right) \\
& \times V_{10}+\frac{A_{0} A_{0}^{\prime} v_{1}}{B_{0}}+\left(\frac{2 B_{0}^{\prime}}{B_{0}}+\frac{B_{0}^{2} r^{2} A_{0} A_{0}^{\prime}}{\Delta_{0}}\right. \\
& \left.+\frac{C_{0}^{\prime}}{C_{0}}+\frac{2 r A_{0}^{2} B_{0}^{2}}{\Delta_{0}}+\frac{2 r^{2} A_{0}^{2} B_{0} B_{0}^{\prime}}{\Delta_{0}}\right) v_{2} \\
& +V_{20}\left\{2\left(\frac{b}{B_{0}}\right)^{\prime}+\frac{A_{0} B_{0}^{2} r^{2} A_{0}^{\prime}}{\Delta_{0}}\left(\frac{a}{A_{0}}+\frac{a^{\prime}}{A_{0}^{\prime}}\right.\right. \\
& \left.+\frac{2 b}{B_{0}}-\frac{d}{\Delta_{0}}\right)+\frac{2 r A_{0}^{2} B_{0}^{2}}{\Delta_{0}}\left(\frac{2 b}{B_{0}}+\frac{2 a}{A_{0}}-\frac{d}{\Delta_{0}}\right) \\
& \left.+\frac{2 r A_{0}^{2} B_{0}^{2}}{\Delta_{0}}\left(\frac{2 a}{A_{0}}+\frac{b^{\prime}}{B_{0}^{\prime}}+\frac{b}{B_{0}}-\frac{d}{\Delta_{0}}\right)+\left(\frac{c}{C_{0}}\right)^{\prime}\right\} \\
& +x_{2}\left(\frac{3 B_{0 \theta}}{B_{0}}+\frac{r B_{0}^{2} A_{0} A_{0 \theta}}{\Delta_{0}}+\frac{r^{2} A_{0}^{2} B_{0} B_{0 \theta}}{\Delta_{0}}+\frac{C_{0 \theta}}{C_{0}}\right) \\
& +X_{20}\left\{3\left(\frac{b}{B_{0}}\right)_{\theta}+\frac{r^{2} A_{0} A_{0 \theta} B_{0}^{2}}{\Delta_{0}}\right. \\
& \times\left(\frac{a}{A_{0}}+\frac{a_{\theta}}{A_{0 \theta}}+\frac{2 b}{B_{0}}-\frac{d}{\Delta_{0}}\right)+\left(\frac{c}{C_{0}}\right)_{\theta} \\
& +\frac{r^{2} A_{0}^{2} B_{0} B_{0 \theta}}{\Delta_{0}}\left(\frac{2 a}{A_{0}}+\frac{b_{\theta}}{B_{\theta}}\right. \\
& \left.\left.+\frac{b}{B_{0}}-\frac{d}{\Delta_{0}}\right)\right\}-v_{3}\left(r+\frac{r^{2} B_{0}^{\prime}}{B_{0}}\right)-r^{2} v_{3}\left(\frac{b}{B_{0}}\right)^{\prime} \\
& -v_{4} \frac{C_{0} C_{0}^{\prime}}{B_{0}^{2}}-\frac{C_{0} C_{0}^{\prime}}{B_{0}^{2}} V_{40}\left(\frac{c^{\prime}}{C_{0}^{\prime}}+\frac{c}{C_{0}}-\frac{2 b}{B_{0}}\right) \\
& -\omega X_{10}\left[1+\left(\frac{3 b}{B_{0}}+\frac{a r^{2} A_{0} B_{0}^{2}}{\Delta_{0}}+\frac{c}{C_{0}}\right)\right] \text {. }
\end{aligned}
$$

\section{References}

1. D. Pietrobon, A. Balbi, D. Marinucci, Phys. Rev. D 74, 043524 (2006)

2. T. Giannantonio et al., Phys. Rev. D 74, 063520 (2006)

3. A.G. Riess et al., Astrophys. J. 659, 98 (2007)

4. S. Capozziello, Int. J. Mod. Phys. D 11, 483 (2002)

5. S. Nojiri, S.D. Odintsov, Phys. Rep. 505, 59 (2011)

6. L. Herrera, N.O. Santos, Phys. Rep. 286, 53 (1997)

7. A. Di Prisco, L. Herrera, V. Varela, Gen. Relativ. Gravit. 29, 1239 (1997)

8. M. Sharif, M.Z. Bhatti, Gen. Relativ. Gravit. 44, 2811 (2012)

9. M. Sharif, M.Z. Bhatti, Int. J. Mod. Phys. D 23, 1450085 (2014)

10. M. Sharif, M.Z. Bhatti, Mod. Phys. Lett. A 29, 1450165 (2014)

11. M. Sharif, M.Z. Bhatti, Mod. Phys. Lett. A 29, 1450094 (2014)

12. M. Sharif, M.Z. Bhatti, Mod. Phys. Lett. A 29, 1450129 (2014)

13. M. Sharif, Z. Yousaf, Phys. Rev. D 88, 024020 (2013)

14. M. Sharif, Z. Yousaf, Mon. Not. R. Astron. Soc. 440, 3479 (2014)

15. M. Sharif, Z. Yousaf, Astropart. Phys. 56, 19 (2014)
16. J.M. Sunzu, S.D. Maharaj, S. Ray, Astrophys. Space Sci. 352, 719 (2014)

17. M. Sharif, Z. Yousaf, Eur. Phys. J. C 75, 58 (2015)

18. M. Sharif, Z. Yousaf, Gen. Relativ. Gravi. 47, 48 (2015)

19. M. Sharif, Z. Yousaf, Astrophys. Space Sci. 357, 49 (2015)

20. L. Herrera, W. Barreto, J. Carot, A. Di Prisco, Class. Quantum Grav. 24, 2645 (2007)

21. W.B. Bonnor, Phys. Lett. A 158, 23 (1991)

22. M. Korunur, M. Saltı, O. Aydogdu, Eur. Phys. J. C 50, 101 (2007)

23. R. Li, Eur. Phys. J. C 73, 2274 (2013)

24. L. Herrera, A. Di Prisco, J. Ibáñez, J. Ospino, Phys. Rev. D 89, 084034 (2014)

25. S. Chandrasekhar, Astrophys. J. 140, 417 (1964)

26. L. Herrera, N.O. Santos, G. Le Denmat, Mon. Not. R. Astron. Soc. 237, 257 (1989)

27. R. Chan, L. Herrera, N.O. Santos, Mon. Not. R. Astron. Soc. 265, 533 (1993)

28. R. Chan, L. Herrera, N.O. Santos, Mon. Not. R. Astron. Soc. 267, 637 (1994)

29. R. Chan, Mon. Not. R. Astron. Soc. 316, 588 (2000)

30. R.G. Cai, Phys. Rev. D 65, 084014 (2002)

31. K. Bamba, S. Nojiri, S.D. Odintsov, Phys. Lett. B 698, 451 (2011)

32. Y.S. Myung, T. Moon, E.J. Son, Phys. Rev. D 83, 124009 (2011)

33. T. Moon, Y.S. Myung, E.J. Son, Eur. Phys. J. C 71, 1777 (2011)

34. S. Capozziello, M. De Laurentis, I. De Martino, M. Formisano, S.D. Odintsov, Phys. Rev. D 85, 044022 (2012)

35. De Laurentis, M., Capozziello, S., arXiv:1202.0394

36. A.V. Astashenok, S. Capozziello, S.D. Odintsov, J. Cosmol. Astropart. Phys. 12, 040 (2014)

37. R. Farinelli, M. De Laurentis, S. Capozziello, S.D. Odintsov, Mon. Not. R. Astron. Soc. 440, 2894 (2014)

38. M. Sharif, M.Z. Bhatti, Astropart. Phys. 56, 35 (2014)

39. M. Sharif, Z. Yousaf, J. Cosmol. Astropart. Phys. 06, 019 (2014)

40. L. Herrera, A. Di Prisco, J. Ospino, J. Carot, Phys. Rev. D 91, $024010(2015)$

41. V. Faraoni, S. Nadeau, Phys. Rev. D 72, 124005 (2005)

42. S. Nojiri, S.D. Odintsov, Phys. Rev. D 68, 123512 (2003)

43. S. Capozziello, S. Nojiri, S.D. Odintsov, Phys. Lett. B 632, 597 (2006)

44. A. Borowiec, W. Godłowski, M. Szydłowski, Int. J. Geom. Meth. Mod. Phys. 4, 183 (2007)

45. S. Capozziello, V.F. Cardone, A. Troisi, Mon. Not. R. Astron. Soc. 375, 1423 (2007)

46. Kelleher, A., arXiv:1309.3523

47. M. Sharif, Z. Yousaf, Astrophys. Space Sci. 354, 471 (2014)

48. M. Sharif, Z. Yousaf, Astrophys. Space Sci. 354, 481 (2014)

49. M. Sharif, Z. Yousaf, Astrophys. Space Sci. 355, 317 (2015)

50. T.P. Sotirou, V. Faraoni, Rev. Mod. Phys. 82, 451 (2010)

51. Harrison, B. K., Thorne, K.S., Wakano, M., Wheeler, J. A., Gravitation Theory and Gravitational Collapse (University of Chicago Press, Chicago, 1965)

52. E. Barausse, T.P. Sotiriou, J.C. Miller, Class. Quantum Grav. 25, 062001 (2008)

53. A.V. Astashenok, S. Capozziello, S.D. Odintsov, J. Cosmol. Astropart. Phys. 01, 001 (2015)

54. C.W. Misner, D. Sharp, Phys. Rev. 136, B571 (1964)

55. M. Gleiser, Phys. Rev. D 38, 2376 (1988)

56. J. Páramos, C. Bastos, Phys. Rev. D 86, 103007 (2012)

57. A. Borisov, B. Jain, P. Zhang, Phys. Rev. D 85, 063518 (2012)

58. M. Sharif, M.Z. Bhatti, Astrophys. Space Sci. 352, 883 (2014)

59. A.V. Astashenok, S. Capozziello, S.D. Odintsov, Phys. Rev. D 89, 103509 (2014)

60. A.V. Astashenok, S. Capozziello, S.D. Odintsov, Astrophys. Space Sci. 355, 333 (2015)

61. S. Anathpindika, Pub. Astron. Soc. Aus. 32, e002 (2015) 
62. H. Bondi, M.G.J.B.D. Burg, A.W.K. Metzner, Proc. R. Soc. A 269, 21 (1962)

64. S. Chandrasekhar, Rev. Mod. Phys. 56, 2 (1984)

63. L. Herrera, A. Di Prisco, J. Ospino, J. Carot, Phys. Rev D 91,

65. F. Dosopoulou, C.G. Tsagas, Phys. Rev. D 89, 103519 (2014) $024010(2015)$ 